\title{
A framework for improving error messages in dependently-typed languages
}

https://doi.org/10.1515/comp-2019-0001

Received June 29, 2018; accepted October 31, 2018

\begin{abstract}
Dependently-typed programming languages provide a powerful tool for establishing code correctness. However, it can be hard for newcomers to learn how to employ the advanced type system of such languages effectively. For simply-typed languages, several techniques have been devised to generate helpful error messages and suggestions for the programmer. We adapt these techniques to dependently-typed languages, to facilitate their more widespread adoption. In particular, we modify a higher-order unification algorithm that is used to resolve and type-check implicit arguments. We augment this algorithm with replay graphs, allowing for a global heuristic analysis of a unification problem-set, error-tolerant typing, which allows type-checking to continue after errors are found, and counter-factual unification, which makes error messages less affected by the order in which types are checked. A formalization of our algorithm is presented with an outline of its correctness. We implement replay graphs, and compare the generated error messages to those from existing languages, highlighting the improvements we achieved.
\end{abstract}

Keywords: higher-order unification, type error diagnosis, counter-factual typing, type-inference

\section{Introduction}

Dependent-types enable developers to enforce rich properties of their programs statically. Through the CurryHoward Correspondence, a dependent-type system provides the full power of higher-order logic for proving program correctness.

Notwithstanding, dependent-types have not seen widespread adoption. This may be in part due to their ex-

\footnotetext{
^Corresponding Author: Joseph Eremondi: University of British Columbia, Canada; E-mail: jeremond@cs.ubc.ca

Wouter Swierstra, Jurriaan Hage: Utrecht University, The Netherlands; E-mail: \{w.s.swierstra, j.hage\}@uu.nl
}

perimental nature, as they are still an active subject of research. The type systems that allow them to provide so many guarantees also enforce a very strict type discipline when programming, and this complexity leads to more complex error messages being reported to the programmer. Thus, a large amount of development time is spent responding to error messages, diagnosing errors, and repairing them.

For beginning programmers, these type errors can be a daunting barrier to entry, particularly when the error messages that the compiler provides are uninformative or confusing. Empirical evidence suggests that difficulty reading error messages is correlated with difficulty completing programming tasks [1]. Generating helpful error messages has been well studied for conventional functional languages like Haskell and ML, through projects such as the Helium Haskell compiler [2-4] and counter-factual typing [5]. However, the topic has been largely unexplored for dependent-types.

In this work, we take the first steps towards improving error messages in a dependently-typed language. We focus on a higher-order unification algorithm, which is a key part of a checker for dependent-types. Taking inspiration from techniques for Haskell-style languages, we provide a representation that allows the entire unification problem-set to be considered simultaneously, allowing for heuristics to diagnose error causes and suggest hints to the programmer as to how they can be repaired. Our complete contributions include:

- An overview of problems with current dependentlytyped error messages (Section 2).

- A new type of constraint graph, called a replay graph, which allows for global heuristic analysis of a unification problem, while accounting for the dependencies inherent to dependent typing (Section 6.2).

- A strategy for error-tolerance, allowing type-checking to continue even after an error is found (Section 6.4).

- The theory behind counter-factual unification, which, when a solution for a variable is found, proceeds by solving the unification problem both with and without the solution, allowing for multiple conflicting solutions to be regarded as equally valid (Section 7). 
- The integration of these techniques into an existing unification algorithm, with proofs that they do not affect correctness (Sections 6.5 and 7.5).

- An implementation of type graphs and error-tolerance in a simple dependently-typed language, with a qualitative comparison of the generated error messages to those from existing languages (Section 8). Our implementation is able to generate repair hints and identify the true cause of the error in many cases.

The focus of this work is on developing a general framework in which heuristics can generate error messages, rather than developing the heuristics themselves. However, even with a small set of heuristics, we are able to provide helpful error messages for several classes of errors.

\section{Error reporting: principles and status quo}

To begin, we highlight the goals of error-message generation by providing classes of unsatisfactory error messages, along with simple examples of ill-typed programs in dependently-typed languages generating such messages. We choose examples from Agda [6] and Idris [7], two (relatively) popular dependently-typed languages that rely heavily on unification. We favour these languages over ones such as Coq, since they use a relatively direct style of programming, as opposed to using tactics to automate proof generation. To keep our presentation clear, we take some liberties, abbreviating syntax and reformatting messages in minor ways. A box is used to highlight the part of the code which the message identifies as faulty.

In Section 8, we show the cases in which we improve upon the error messages given by Agda and Idris, establishing that unhelpful messages are not inherent to dependent-types.

\subsection{Error location and cause}

The main goal of a (type) error message is to inform the user which code they must change in order for the program to be well typed. Part of this involves error-message location, reporting one or more source-code locations as a possible cause of an error. When multiple source locations are relevant to the error, we wish to report all locations that provide the programmer with information necessary to make a repair. A simple strategy for this is to re- port all locations involved with an error [8]. Alternatively, heuristics can guess the ideal location, as in Helium [2, 3] and ShErrLoc [9].

Additionally, we would like error messages to indicate the cause of the error: the specific mistake made, whose correction will cause the program to type-check. This typically consists of a high-level, natural language message given to the programmer. Even better is to suggest a repair, giving not only the problem in the code, but the change that must be made to correct it. Helium generates such messages using heuristic analysis [3].

\subsubsection{Multiple error locations}

Consider the following Agda code. The function vecFoldr is a dependently-typed version of the classic foldr: it iteratively applies a function over a list, but the return type of this function may depend on the number of elements processed so far. This allows us to iterate over a vector (i.e. a list with its length indexed in its type) and ensure that the returning list's length is related to the original length in some way. The function doubleHead takes a number and a list and uses cons to append double that number to the head of the list.

Finally, the code shows an application of vecFoldr, folding doubleHead over a list of Booleans. Since the type of list elements, the list length, and the type we are returning are all explicit arguments to vecFol dr, the programmer will often wish to omit these. To do so, they write _ to indicate that the compiler should infer the value of that particular argument. However, in this example, when we try to fold doubleHead over a list containing Booleans, instead of numbers, we get a type error.

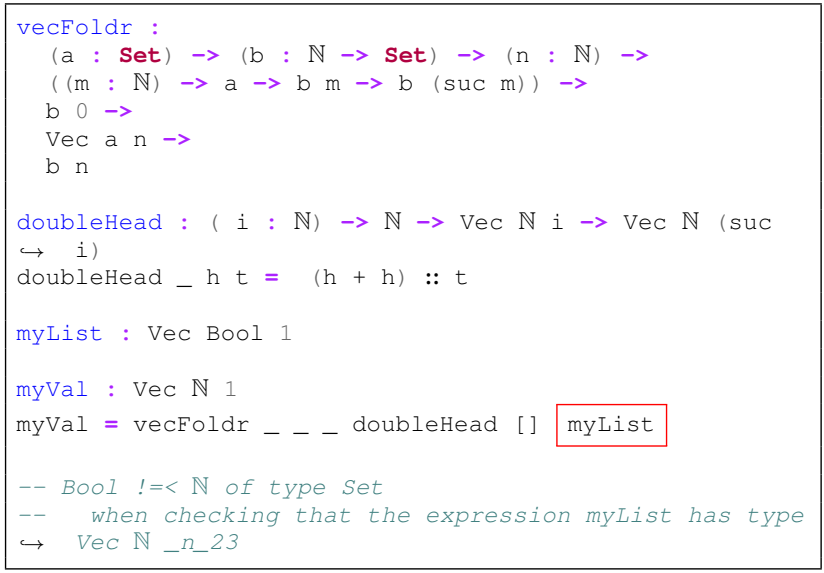

The reported message locates the error at myList, which is partially correct, since it is an argument of the 
wrong type. However, it is missing crucial information, namely that we are expecting a number because of the type of doubleHead. Thus, both locations are relevant to the error. This cause is hidden by the fact that this constraint is carried through the unification variables, induced by the implicit arguments of vecFoldr.

Our framework, conversely, generates the following message:

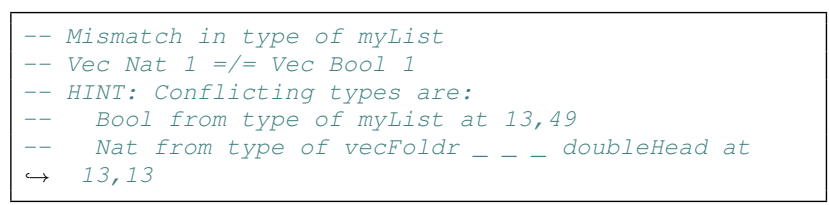

In this message, two conflicting locations are identified, and the user is directed both to myList, and to the application of vecFoldR to doubleHead.

\subsubsection{Repair hints}

In addition to highlighting all relevant locations in the code, we wish for error messages to describe the cause of the error, and to hint at how to fix it. The heuristics we provide are particularly good at identifying the cause of an error and reporting a hint for how to fix it. Consider the following code snippet, where arguments to a polymorphic function are given in the wrong order.

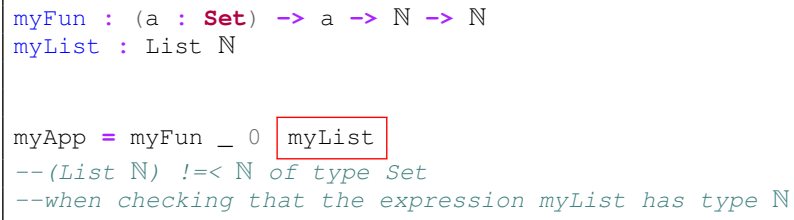

Again, the user is told that the type is wrong, but not why it is wrong. Our heuristics instead generate the following message, which not only reports the error, but gives its cause (arguments in the wrong order) and a possible repair (rearranging the arguments).

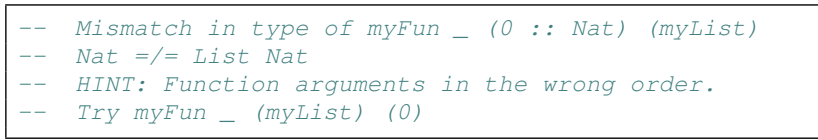

We give this example in more detail, along with several more examples of improvements we achieve, in Section 8.

\subsection{Left-to-right bias}

Depending on the order a program is traversed in a typeinference algorithm, bias can be introduced. Unification variables are generated for expressions and may be assigned a value as the algorithm progresses. If the algorithm attempts to assign another type to the same variable, that type will be marked as incorrect, even if it is the desired value and the initial value was wrong. The order in which the syntax tree is traversed determines which type is assigned to the expression, and which type is viewed as incorrect.

This kind of bias interferes with the goals of error message generation, since both the assignment of an error location, and the reported error cause, depend on the order in which unifications are performed, as opposed to the actual location or cause. This type of bias is particularly problematic when implicit arguments are introduced, since annotations are not always present to express the programmer's intent.

Consider the following Agda code, with corresponding errors:



The correct type is assumed to be the type of the first element of the list, even though switching the types of either list will remove the error. What's more, the reported errors do not change if type annotations are added! We can see a similar bias in Idris:

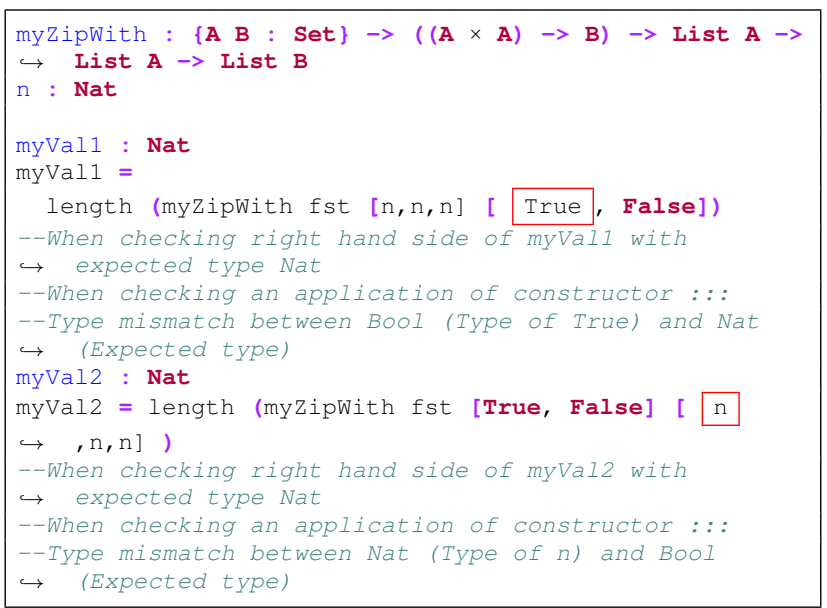


Various approaches from the literature aid in reducing bias. The Helium compiler [2, 3] reduces bias through constraint-based type-checking, and by representing a unification problem as a single graph, allowing for global analysis regardless of the order in which constraints are added. Counter-factual typing [5] reduces bias by also finding the solutions that would have been generated if that constraint had never been added.

Bias introduces additional complexities specific to dependently-typed languages: the ordering in which constraints are solved may affect not only which type is assumed correct, but also which value of an index for a typefamily is assumed correct. Since evaluation occurs during type-checking, the first value chosen as "correct" can affect the result of evaluation, dramatically changing the types involved.

Our contribution uses graph-based checking (Section 6) to reduce bias. However, due to the complexities of dependent-types, not all bias can be eliminated with graph-based checking. We present counter-factual typing (Section 7) as a theoretical way to reduce bias, though our implementation does not yet use this technique.

\subsection{Other unsatisfactory messages}

Here we present additional examples of unsatisfactory messages. Some fall into the categories described but use advanced type features not modeled by our core calculus, and others have messages whose deficiencies are not due to error location or bias. These problems will not be helped by the techniques we present. However, this work is, to the authors' knowledge, the first examining the state of dependently-typed error messages, so we catalogue them here for the sake of completeness, hoping to inspire future work.

\subsubsection{Pattern-matching and rewriting}

Dependent pattern matching is a key feature in dependently-typed languages but is also a source of complexity. With dependent pattern matching, the values matched in the left-hand side influence the expected type of the right-hand side. Additionally, matching on one argument to a function can imply specific values that the others must take.

Here we give a flawed proof of symmetry of equality. The program pattern matches the proof of $x=y$ with $r e f l$, its only possible value, and tries to return refl as a proof that $y=x$. However, refl returns a proof that $x=x$, causing a mismatch.

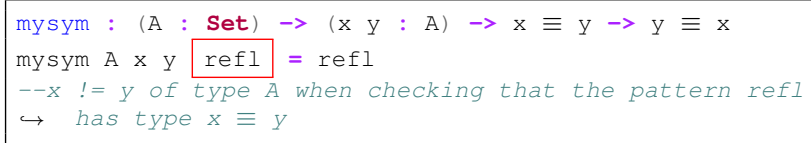

The error informs us that $\mathrm{x} !=\mathrm{y}$, but never tells us why it is expected that the variables be equal, except perhaps by indicating that $r e f l$ is the error location. The true cause of the error is that the pattern match must also specify that $x$ and $y$ are equal, but the user is not informed of this. This can be repaired using Agda's dot patterns.

mysym $A \times \cdot x$ refl $=\operatorname{refl}$

The Idris equivalent faces a similar problem, and while it provides more hints as to the cause, it unhelpfully reports the function name as the location of the error.

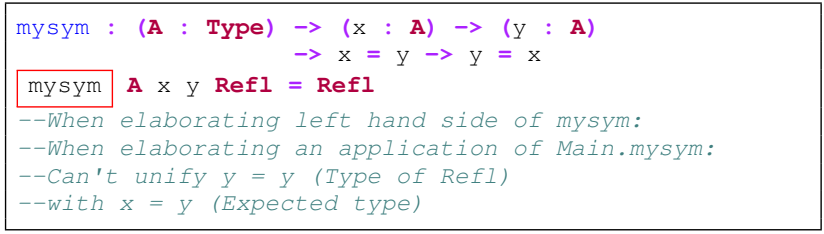

The information given is similarly vague when we rewrite goals using equality proofs. The following code contains nilNeutralR, a proof that appending a list to the empty list does not change it, and tries to show that appending the empty list does not change the length of a list. It uses a rewrite, where an equality is used to replace a term throughout the goal type. However, the equality must be flipped before using it.

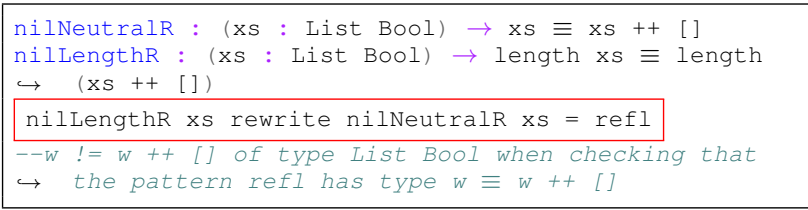

The code can be fixed by using sym nilNeutralR in place of nilNeutralR, but the error message gives no hints that this repair would solve the problem.

\subsubsection{Amechanicity}

Another goal of error messages is to ensure that error messages are amechanical [10]: we want the reported messages 
to avoid leaking internal details of the compiler, and to be rooted in the provided source code.

For instance, many Agda error messages will refer to intermediate metavariables generated by the unification algorithm. Consider the error for the following code, which includes metavariable names that never appear in its source code.

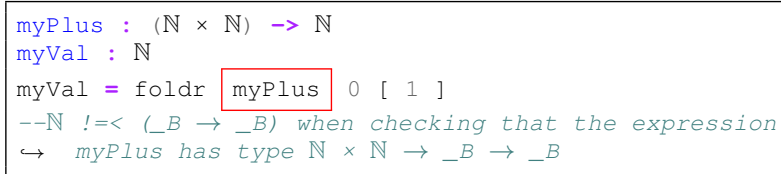

Uninformative messages also occur when a code snippet contains a metavariable which does not have a unique solution. Here, the messages given by Agda contain almost no clues as to the underlying cause.

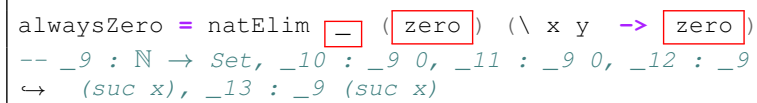

Idris reports a similar message, though a type mismatch is reported, as opposed to an unsolved metavariable:

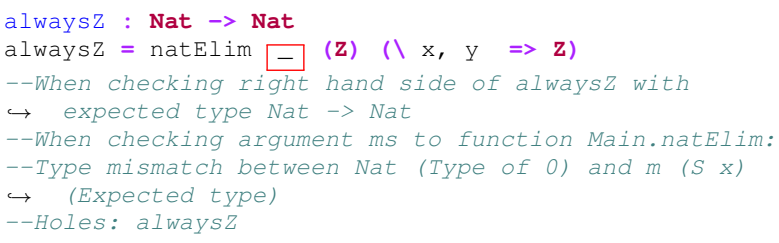

Similarly, if unification ever gets stuck, the user is provided with an unhelpful message, referring to the internal numbers of unification problems, giving no indication of why the problems are blocked. The following example was adapted from an error encountered by an unfortunate user on Reddit [11], who was faced with the internal details of the unification solver while trying to write a simple proof.

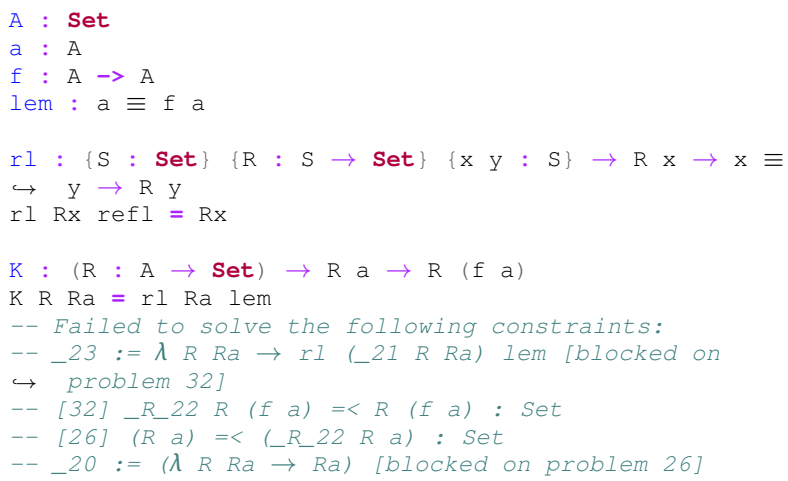

\section{Background}

To describe how to generate quality error messages for dependent-types, we first introduce some prerequisite concepts. We assume familiarity with the basics of type theory and dependently-typed languages, as well as with basic concepts from graph theory, such as connected components.

\subsection{Preliminaries}

We focus on dependently-typed languages that combine terms and types into a single syntactic category, with a type of types, denoted Set.

We use the notation $\bar{X}_{i}^{i}$ to represent a sequence of $i$ elements from whatever set $X$ ranges over.

We denote the operation of substituting all free occurrences of $x$ with $Y$ in $Z$ as $[x \Leftrightarrow Y] Z$. Here $x$ may be a program variable or metavariable, $Y$ is a term, and $Z$ may be any structure that binds these, such as terms, types, contexts, metacontexts, etc. We sometimes find it convenient to combine substitution and evaluation to $\beta$-normal $\eta$-long form into a single operation, denoted $[x \vec{\Leftrightarrow} Y] Z$. This is conceptually similar to hereditary substitution [12].

If $X$ is a term, context, etc. then we denote the set of free program variables in $X$ as $\operatorname{FV}(X)$, and the set of all free and bound variables in $X$ as $\operatorname{vars}(X)$. The set of free metavariables in $X$ is denoted by $\operatorname{FMV}(X)$.

For rewrite rules, e.g., reduction $\rightarrow$ and metacontextsolving $\mapsto$, we use the symbol ${ }^{\star}$ (e.g. $\rightarrow^{\star}$, $\mapsto^{\star}$ ) to denote the reflexive and transitive closure of these relations.

\subsection{Definitional equality}

Throughout this paper, we will be searching for unifiers that cause two terms to be equal. It's important to note that deciding extensional equality, whether two terms always behave the same way, is not possible. We instead use definitional equality [13], denoted $s={ }_{\beta \delta \eta} t$, which asserts that two terms are identical up to $\beta$-reduction, $\eta$-expansion, substitution of top-level definitions $\delta$, and bound variable renaming.

\subsection{Higher-order unification}

We define the higher-order unification problem for a programming language as follows. We begin with a set $V=$ $\left\{\alpha_{1}, \ldots, \alpha_{n}\right\}$ of metavariables, and a set of problems, of the 
following form:

$$
\forall x_{1}: T_{1}, \ldots, x_{k}: T_{k} . s: S \equiv t: T
$$

where

$$
F V\left(s, t, S, T, T_{1}, \ldots, T_{k}\right) \subseteq\left\{x_{1}, \ldots, x_{k}\right\}
$$

and

$\mathrm{FMV}(s), \operatorname{FMV}(t) \subseteq V$

When it is clear to do so, we omit the types from our notation for problems.

The goal of higher-order unification is to find an assignment of closed terms $t_{1}, \ldots, t_{n}$ to metavariables $\alpha_{1}, \ldots, \alpha_{n}$, such that for each problem:

$$
\forall{\overline{x_{i}}: T_{i}}_{i}^{i} s: S \equiv t: T
$$

and for every well-typed sequence of values ${\overline{v_{i}: T_{i}}}^{i}$, we have:

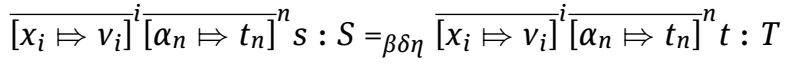

Additionally, solutions should satisfy:

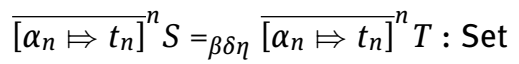

That is, we wish to find an assignment that causes both sides of each equivalence to become well typed and definitionally equal.

Higher-order unification is in general undecidable, and for many instances there is no unique solution which generalizes all other solutions. However, Miller [14] identified a sub-problem which is decidable and admits mostgeneral unifiers: the pattern fragment, where metavariables can only be applied to distinct bound variables [15]. Essentially, the pattern fragment allows for higher-order constraints of the form $\forall x_{1} \ldots x_{n} . \alpha x_{1} \ldots x_{n} \equiv t$, since these can be solved by $\alpha:=\lambda x_{1} \ldots x_{n} . t$.

Unification is used in dependently-typed languages for two main purposes. The first is dependent pattern matching. Here, the type of the value being matched upon is unified with the constructor's return type, and the constructor's argument types are unified with the types of the matched variables. For example, when defining:

$$
\text { head : ( } \mathrm{V} \text { : Vec A }(\operatorname{SucC} \mathrm{n})) \rightarrow \mathrm{A}
$$

the constructor null has return type $\operatorname{Vec} A$, so when we pattern match on $v$, the case for null is omitted, since we cannot unify $\mathrm{Vec} A \mathrm{~A}$ with

Vec A (Succ $\mathrm{n}$ ). Conversely, the unification succeeds for the cons constructor. A detailed account is given by Norell [16].

In this work, however, we focus on a second use: unification of program metavariables. These can be used in place of expressions, when there is only one possible value the expression can have in order to type-check correctly. In Agda and Idris, metavariables are denoted with the underscore character _. We will likewise use this notation in our example programs.

Program metavariables can be used in the implementation of implicit arguments, which allow the programmer to call a function with metavariables automatically inserted for specific missing arguments. These are crucial for using polymorphism without notational overhead in dependently-typed languages, since many use System-F style explicit instantiation of type variables. For example, the identity function has type ( $T$ : Set) $\rightarrow T \rightarrow T$, and it must be first applied to a type before it can be applied to a value, such as in id Nat 0 . To reduce the burden on the programmer, the parameter $T$ can be made implicit: at compile time it is replaced with a metavariable, and its value is found through unification.

\section{The base language}

To showcase our techniques for generating error messages, we set the stage with a dependently-typed core calculus, and a conventional higher-order unification algorithm. A noteworthy aspect of our presentation is that we use a single relation to capture both type-checking and finding the normal form of a term. Otherwise, the syntax and semantics are fairly standard, and serve primarily to provide the vocabulary with which we can describe our contributions in later sections.

\subsection{Syntax}

The syntax for our language is presented in Figure 1. As a dependently-typed language, types and terms inhabit a single syntactic category. We have $\Pi S T$ for functions that, when given an argument $x$ of type $S$, return a value of type $T x$. The pair type $\Sigma S T$ is similar: if the first element is $x$ of type $S$, the second element has type $T x$. Our language features no explicit polymorphism, but the familiar type $\forall X . T[X]$ can be simulated using $\Pi$ Set $(\lambda X . T[X])$. We use $S \rightarrow T$ as a shorthand for $\Pi S(\lambda x . T)$ where $x$ is not free in $T$.

Many presentations of dependent-types use a form $\Pi(x: S) T$ or $(x: S) \rightarrow T$ for the dependent function type. Instead, function codomains and pair secondelement types are encoded as $\lambda$-terms in our system, since this will allow us to express equalities between them without worrying about the specific bound names. The above 


\section{Syntax \\ Terms: \\ $s, t, S, T::=$ \\ $x$ (Program Variable) \\ $t: T$ (Type Annotation) \\ Set (Type of types) \\ $t \cdot{\overline{e_{i}}}^{i} \quad$ (Elimination) \\ $\Pi S T$ (Function type) \\ $\Sigma S T \quad$ (Pair type) \\ $\lambda x . t \quad$ (Functions) \\ I $\left(t_{1}, t_{2}\right) \quad$ (Pairs)}

Eliminators:

$$
\begin{aligned}
& d, e::=\quad \text { funElim } t_{\text {arg }} \quad \text { (Function app.) } \\
& \pi_{1} \quad \text { (Pair first proj.) } \\
& \pi_{2} \text { (Pair second proj.) }
\end{aligned}
$$

Neutral term heads:

$$
\begin{array}{cl}
h::= & x \quad \text { (Variable head) } \\
\mid & \alpha \quad \text { (Metavariable head) }
\end{array}
$$

Neutral Terms:

$u, v, U, V::=\ldots$

$$
h \cdot{\overline{e_{i}}}^{i}
$$

(Neutral elimination)

$$
\begin{aligned}
\mathrm{E}::= & \square|t: \mathrm{E}| \mathrm{E}: V|t \mathrm{E}| \mathrm{E} v\left|\pi_{1} \mathrm{E}\right| \pi_{2} \mathrm{E} \\
& |\Pi \mathrm{E} T| \Pi V \mathrm{E}|\Sigma \mathrm{E} T| \Sigma V \mathrm{E} \mid(\mathrm{E}, t)
\end{aligned}
$$

Reduction semantics: (phrased in traditional notation)

$$
\begin{aligned}
(\lambda x . s) t & \rightarrow[x \mapsto t] s \\
\pi_{1}(s, t) & \rightarrow s \\
\pi_{2}(s, t) & \rightarrow t \\
\mathrm{E}[t] & \rightarrow \mathrm{E}\left[t^{\prime}\right] \quad \text { if } t \rightarrow t^{\prime}
\end{aligned}
$$

Figure 1: Base language: syntax and semantics.

type would be represented as $\Pi S(\lambda x . T)$, explicitly binding the argument value in a $\lambda$. Our $\Sigma$ types are represented similarly.

In our syntax, elimination of terms is represented using spine form [17]: where a sequence of eliminators is applied to a term. This gives us easy access to the head of a sequence of applications, which will prove useful in our unification algorithm. A neutral term is a spine term whose head is a variable or metavariable, named as such because they cannot be reduced. For readability, we will often write applications and projections using the traditional notation. For example, we write $x \cdot\left(\right.$ funElim $\left.f, \pi_{1}\right)$ as $\pi_{1}(f x)$ when convenient. Similarly, we will be flexible with our notation, sometimes using eliminators where a term is expected, such as on both sides of a unification problem.

The set of values is very similar to the set of terms, with the restriction that eliminators may only be applied to variables or metavariables, that is, all spine terms must be neutral terms.

In our implementation, we supported a few inductive types, such as Nat, Eq, and Vec, with eliminators in place of explicit recursion. While we omit these from our model for brevity (as they do not significantly affect our results), we will use them in our examples and evaluations, to provide realistic examples of dependently-typed code.

\subsubsection{Semantics}

The semantics of our language, shown in Figure 1, match those of a standard call-by-name lambda calculus. Function applications are evaluated through substitution, and projections extract the relevant part from a pair. Context rules allow for evaluation deep within terms. The impredicative assumption that Set : Set means that not all terms have a normal form, but we nevertheless choose such a system for the sake of simplicity. Solutions to this problem include separating types from kinds [18], or a hierarchy of universes [19]. Such solutions are orthogonal to the work we present here.

\subsubsection{Declarative typing}

In Figure 2, we define our language's type system. In the absence of metavariables, the type rules for our language are fairly standard. They are not quite syntax directed, but can easily be made so [20, 21].

We follow Löh et al. [20] and use a bidirectional type system, distinguishing type-synthesis judgments $\Rightarrow$, where types are viewed as output, from type-checking judgments $\Leftarrow$, where types are viewed as input. Similar to Gundry [21], we use a single relation to define both the typing of terms and their $\beta$-reduced, $\eta$-long normal forms. We write $\Gamma \vdash t \rightsquigarrow v \Leftarrow T$ to denote that a term $t$ has normal form $v$ and checks against type $T$, with a similar definition for synthesis. This allows for an easy formulation of definitional equality. It will also prove useful when defining 


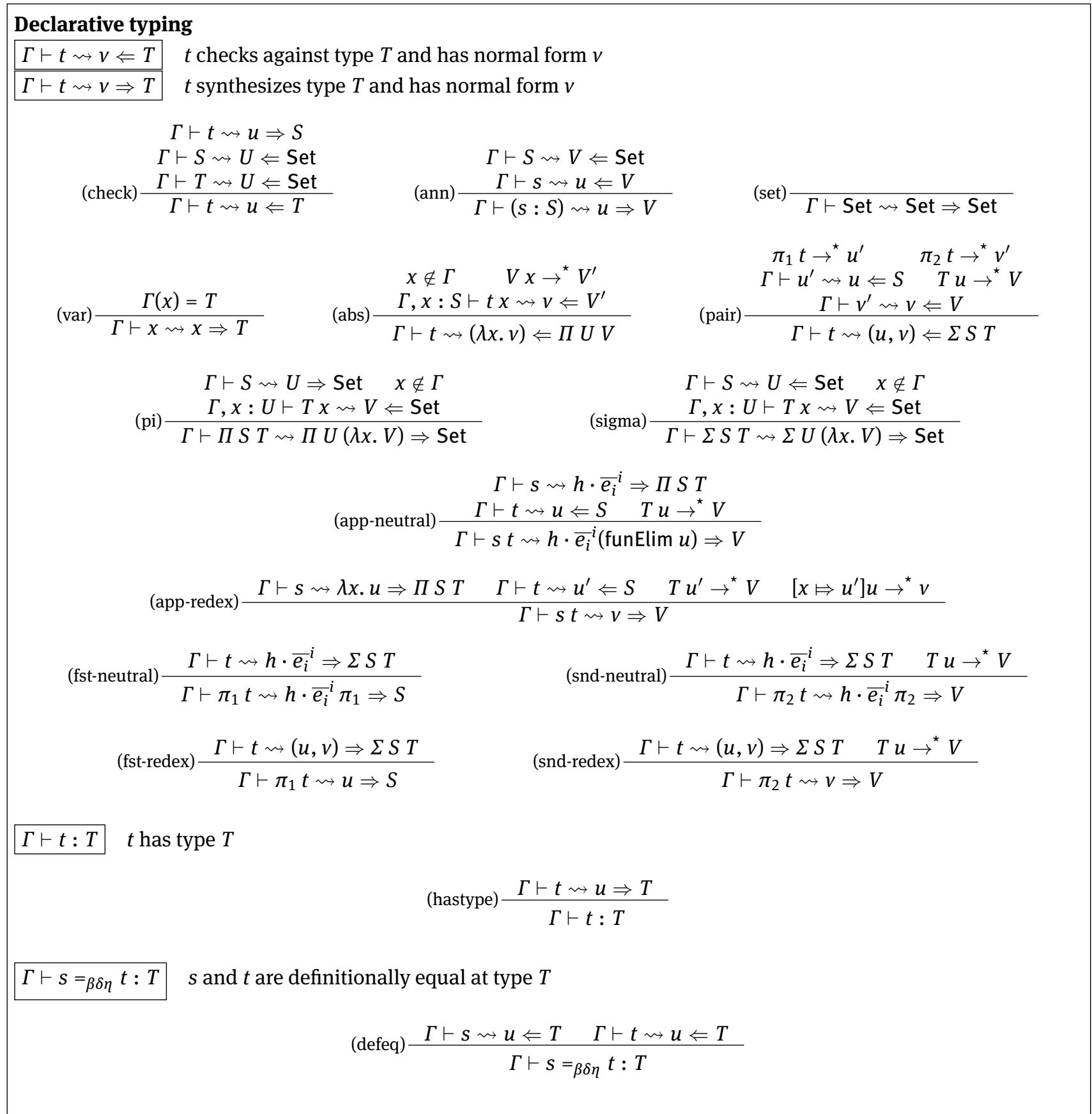

Figure 2: Declarative typing.

error-tolerant typing (Section 6.4), since we can produce normal forms for terms where ill-typed subterms are replaced with $\perp$. The complete rules are defined in Figure 2 .

The (check) and (ann) rules are standard for bidirectional systems: (check) allows us to check a term against any type with the same normal form as its synthesized type, and (ann) allows us to synthesize a type for an annotated term, provided it checks against that type. The (set) rule encodes impredicativity, and (var) simply looks up variable types in the environment.

The (pi) and (sigma) rules perform kind checking: since types and terms overlap, we must make sure that types do not refer to ill-typed terms. We ensure that the codomains of functions are in $\eta$-long form by substituting a fresh variable for their argument, evaluating their bodies to a normal form, and abstracting over the variable. We perform a similar process for $\Sigma$ types. 
The (abs) and (pair) rules type introduction forms, but are checking rules rather than synthesis rules, since we require information from annotations in order to type them. As with (pi) and (sigma), we check abstractions by substituting a fresh variable in for the argument, checking the body, and re-wrapping in a $\lambda$ to produce an $\eta$-long result. To check a pair construction, we check its first element, then compute the expected type of the second element by substituting the value of the first into the function provided by the $\Sigma$ type, then evaluating. To make pairs $\eta$-long, we evaluate the normal forms of the first and second elements and produce the pair containing them as a normal form.

The elimination rules each come in two forms: one for redexes, and one for neutral terms, although the rules differ only in the normal forms produced. To type an application, we synthesize the function type, check the argument against its expected type, and compute the return type by substituting the argument value into the codomain type and evaluating. Typing a pair's first projection is trivial. To type a second projection, we synthesize the type of the pair, then instantiate with the value of the first projection. To compute normal forms, we perform reduction in the redex case, and add the eliminator to the spine in the neutral case.

At the bottom of Figure 2, we provide two relations for convenience. One is simply type-checking that discards normal forms. The second gives the meaning of definitional equality: two terms are definitionally equal at a given type if they check against that type with the same normal form.

\subsubsection{Metavariables and constraint generation}

To introduce metavariables into our language, we must first store their typing information in metacontexts, defined in Figure 3. These can contain declarations, where a metavariable's name and type are given, problems, which constrain two terms to be equal relative to a set of universally quantified variables, and assignments, which give concrete solutions for metavariables. Assignments are not generated during type-checking but will be created and used during unification.

The addition of metavariables requires that each declarative rule also take a metacontext $\Delta$ alongside the telescope $\Gamma$. However, these are always simply passed through, except in the typing rule for metavariables. We provide this rule, along with the syntax for metacontexts, in Figure 3.

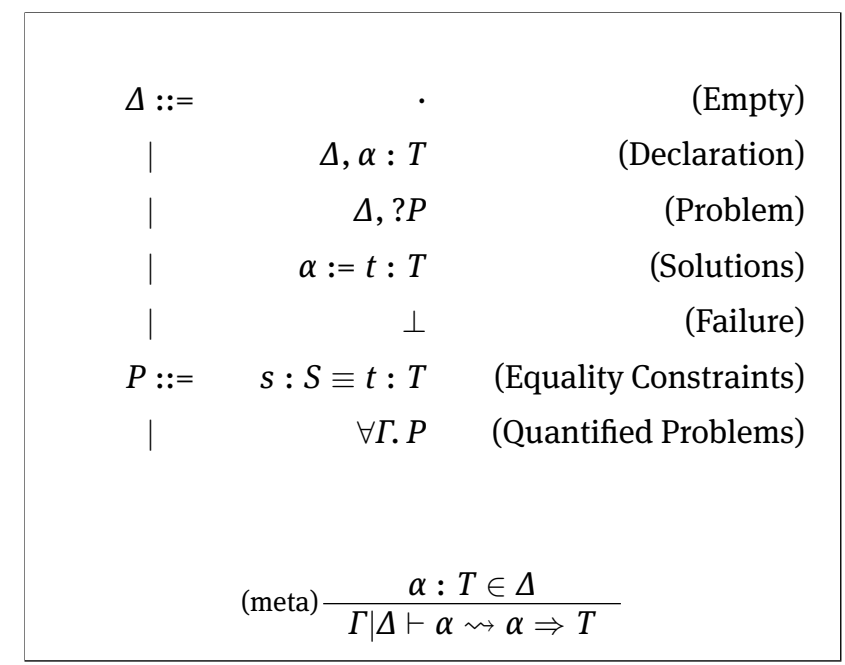

Figure 3: Metacontext syntax and typing.

Once we add metavariables to our language, we require global inference in order to type-check our programs. Both programs and types may contain metavariables that must be solved in order to complete type-checking.

To check a program with metavariables, we follow the standard approach for turning a type-checking algorithm into a constraint-based one: each time a rule patternmatches on an input, we replace that with an equality constraint. Constraint-based type-checking takes a metacontext as input, and produces as output one that contains the constraints that must be satisfied for the program to be well typed. This is then passed to a separate unification solving procedure. A full algorithm is provided by Norell [16], ensuring type-safety through guarded constants.

\section{Solving higher-order unification: a primer}

The main contribution of this work is a series of modifications to higher-order unification which facilitate the generation of quality error messages. However, to discuss modifications, we must first give the intuition behind higherorder unification. Here we present a higher-order unification algorithm, which we refer to as the unmodified algorithm. We highlight the intuition behind it, particularly the parts that we will modify later on. The rules we present are based on those created by Gundry and McBride [22]. We chose this algorithm for its (relative) simplicity and for its ease of implementation, as higher-order unification tends 
to be complex and detail-heavy. Their algorithm is in turn based on that of Abel and Pientka [15], which is somewhat cleaner theoretically, but less easily implemented.

\subsection{Occurrences}

With unification, we will often use the following terminology to describe the occurrences of terms, variables, metavariables, etc. [15]. An occurrence is flexible if it occurs as an argument to a metavariable and is rigid otherwise. If a variable occurs flexibly before a solution from unification is applied, it may not occur at all after the solution is applied, if the metavariable solution is a function that ignores its argument. We say that an occurrence is strongrigid if it is not an argument to a variable or metavariable, meaning that no substitution can eliminate it.

\subsection{Unification as rewriting}

Our algorithm is presented as a non-deterministic set of rules for rewriting metacontexts, which can be seen in Figure 4. We write $\Delta \mapsto \Delta^{\prime}$ if we can rewrite $\Delta$ into $\Delta^{\prime}$ in a single step, and use $\mapsto^{\star}$ to represent the reflexive symmetric transitive closure of the rewrite relation. Details for making the rewrite rules syntax directed, as well as a sample implementation, are given by Gundry and McBride [22].

The algorithm we present is dynamic: when it encounters problems outside of the pattern fragment (as defined in Section 3.3), it simply defers their solutions. Since other problems may refer to the same metavariables, substituting in their solutions may move other problems into the pattern fragment. Because of this dynamic property, our algorithm may become stuck if no failure has been encountered, but no remaining problems are in the pattern fragment. We use the following terminology to refer to the different states a metacontext can take.

Definition 1. We say that a metacontext $\Delta$ is solved if it contains no problems. A metacontext is stuck if it is not solved, but no progress can be made. A metacontext is said to be final if it is solved, stuck, or $\perp$.

Our rules can be grouped into three broad categories:

- Solution rules: When there is only one possible solution $t^{\prime}$ to a problem $\forall \Gamma . s: T \equiv t: T$ containing a metavariable $\alpha$, then we rewrite it to be $\alpha:=t^{\prime}: T$. Similarly, reflexive equations can be deleted.
- Failure rules: These rules identify problems that have no solution, or that cannot possibly be moved into the pattern fragment.

- Simplification rules: These rules are used to manipulate individual problems or parts of the metacontext, progressing towards a form that can be handled by the other rules. For example, metacontexts can be permuted in dependency-respecting ways. Likewise, to propagate our solutions, when $\alpha:=t: T$ occurs in a metacontext, we replace $\alpha$ with $t$ everywhere else. Other rules perform type-directed manipulation of context. For instance, problems comparing functions $f$, $g$ of $\Pi$-types use $\eta$-expansion to instead compare the function bodies.

In Figure 4 we define the solution and failure rules and explain them in the following sections. Simplification rules are critical for applying unification in a practical setting, since they greatly increase the number of problems that can be solved. However, their effect is orthogonal to our changes, so we omit these rules.

\subsubsection{Solution rules}

\section{Rigid equations}

All expressions, other than spine forms with metavariable heads, have rigid heads. The rigid head can be a program variable at the head of a spine, or the $\Pi, \Sigma$ or Set type constructors. We have a rigid-rigid matching when unifying two terms with rigid heads.

To match two expressions with the same variable head, we can generate equalities between their spines, as seen in the (rigid-spine) rule. Similarly, matching two types with the $\Sigma$ or $\Pi$ constructors generates matches between their arguments. For example, $\Pi \alpha T \equiv \Pi \beta T^{\prime}$ is decomposed into $\alpha \equiv \beta, T \equiv T^{\prime}$.

We use our notation flexibly, matching eliminators as well as terms, performing similar decomposition with the eliminator constructors as with term constructors.

\section{Inversion and intersection}

A problem is in the pattern fragment if any elimination with a metavariable head is applied to a spine of distinct program variables. We make progress when one or both sides of an equation are an elimination of this form, since these equations have unique solutions.

A flex-rigid equation is one where one side of the equation is a spine-form term with a metavariable head. (Note that the spine may be empty, for example, with constraints 
$\Delta \mapsto \Delta^{\prime}$

Solution rules

$$
\begin{aligned}
& \Delta, ? \forall \Gamma .(t: T) \equiv\left(t^{\prime}: T^{\prime}\right) \mapsto \Delta \quad \text { if } \Gamma \mid \Delta \vdash T==_{\beta \delta \eta} T^{\prime}: \text { Set and } \Gamma \mid \Delta \vdash t=_{\beta \delta \eta} t^{\prime}: T \\
& \Delta, \forall \Gamma \text {. (П } A B: \text { Set }) \equiv(\Pi S T: \text { Set }) \mapsto \Delta, \forall \Gamma \text {. (A: Set }) \equiv(B: \text { Set }) \wedge(B: A \rightarrow \text { Set }) \equiv(T: S \rightarrow \text { Set }) \\
& \text { (reflexivity) } \\
& \Delta, \forall \Gamma \text {. ( } \Sigma A B: \text { Set }) \equiv(\Sigma S T: \text { Set }) \mapsto \Delta, \forall \Gamma \text {. (A: Set }) \equiv(B: \text { Set }) \wedge(B: A \rightarrow \text { Set }) \equiv(T: S \rightarrow \text { Set }) \text { (rigid-sigma }) \\
& \Delta, \forall \Gamma . x{\overline{d_{i}}}^{i} \equiv x{\overline{e_{i}}}^{i} \mapsto \Delta, \overline{\forall \Gamma \cdot d_{i} \equiv e_{i}} \\
& \text { (rigid-spine) } \\
& \Delta, \alpha: T, \Delta^{\prime}, ? \forall \Gamma . \alpha{\overline{x_{i}}}^{i} \equiv t \mapsto \Delta, \Delta^{\prime}, \alpha:=\lambda{\overline{x_{i}}}^{i} . t: T \\
& \text { (inversion) } \\
& \text { if }{\overline{x_{i}}}^{i} \text { is linear on } \mathrm{FV}(t), \alpha \notin \operatorname{FMV}\left(\Delta^{\prime}, t\right) \text { and } \cdot \mid \Delta, \Delta^{\prime} \vdash \lambda{\overline{x_{i}}}^{i} \cdot t: T \\
& \Delta, \alpha: \Pi \Phi . T, \Delta^{\prime}, ? \forall \Gamma . \alpha{\overline{x_{i}}}^{i} \equiv \alpha{\overline{y_{i}}}^{i} \mapsto \Delta, \Delta^{\prime}, \beta: \Pi \Phi . T, \alpha:=\lambda \Phi . \beta \Psi: \Pi \Phi . T
\end{aligned}
$$

(intersection)

if ${\overline{x_{i}}}^{i}$ agrees with ${\overline{y_{i}}}^{i}$ on $\Psi \subset \Phi$ and $\operatorname{FV}(T) \subset \operatorname{vars}(\Psi)$

Failure rules

$$
\begin{array}{rlr}
\Delta, \forall \Gamma .(s: T) \equiv(t: T) \mapsto \perp & \text { if } s \text { and } t \text { have different rigid heads } \\
\Delta, \alpha: T, \Delta^{\prime}, ? \forall \Gamma . \alpha \bar{x}_{i}^{i} \equiv t \mapsto \perp & \text { if } \alpha \text { occurs rigid-strong in } t \\
\Delta, ? \forall \Gamma . \alpha{\overline{d_{i}}}^{i} \equiv t \mapsto \perp & \text { if } \mathrm{FV}^{\text {rigid }}(t) \not \subset \mathrm{FV}\left({\overline{d_{i}}}^{i}\right)
\end{array}
$$

Figure 4: Higher-order unification, adapted from Gundry and McBride [22].

of the form $\alpha \equiv t$ ). Similarly, a flex-flex equation is one where both sides of the equation are spine-form terms with a metavariable head.

To solve a flex-rigid equation $\alpha{\overline{x_{n}}}^{n} \equiv t$, if ${\overline{x_{n}}}^{n}$ is a sequence of distinct bound variables (i.e. the problem is in the pattern fragment), we generate a solution $\alpha$ := $\lambda{\overline{x_{n}}}^{n}$.t. This process is known as inversion and can be seen in the (inversion) rule. Flex-flex equations with different metavariable heads can be solved in the same way. If the problem is not in the pattern fragment, we wait, hoping that application of other rules will simplify it to be in the pattern fragment.

A special case, however, occurs when our problem is $\alpha \cdot{\overline{x_{i}}}^{i} \equiv \alpha \cdot{\overline{y_{i}}}^{i}$, that is, both equations have the same metavariable head. Here we instead solve by intersection: a fresh metavariable $\beta$ is declared, and we generate the solution $\alpha:=\lambda x_{1} \ldots x_{n} . \beta z_{1} \ldots z_{k}$, where $\left\{z_{1}, \ldots, z_{k}\right\}=$ $\left\{x_{i} \mid x_{i}=y_{i}\right\}$. That is, the solution to such a problem can only refer to the variables shared by both sides of the equation: $\alpha$ is defined as a function which ignores all arguments on which $x_{i}$ and $y_{i}$ disagree. This process is formalized in the (intersection) rule.

\subsubsection{Failure rules}

\section{Rigid-rigid mismatch}

All constructors in our language are injective. Thus, if two expressions have different rigid heads, they cannot be unified, and we fail with the (rigid-fail) rule. Note that injectivity may not apply in univalent settings, where not all constructors are injective [23].

\section{Occurs-check failure}

When solving for a flex-rigid or flex-flex equation $\alpha \bar{e}_{i}^{i} \equiv t$, we must ensure there are no rigid-strong occurrences of $\alpha$ in $t$. If such an occurrence exists, then there is no finite term satisfying the equation, and we fail with the (occurscheck) rule. We only fail for rigid strong occurrences since other occurrences may disappear when solutions for other variables are found.

For example, there is no finite solution to $\alpha \equiv \Pi \alpha \beta$, and our occurs check would fail in this case.

\section{Pruning}

Before solving a flex-rigid or flex-flex equation, we prune the equations. Similar to the occurs check, when solving $\forall \Gamma . \alpha \cdot{\overline{e_{i}}}^{i} \equiv t$, we need to ensure all variables in $t$ (which 
are bound by the $\forall \Gamma$ ) also occur in the spine ${\overline{e_{i}}}^{i}$, since only those variables can be incorporated into a solution for $\alpha$.

If any are not in the spine, but are arguments to a metavariable within $t$, we can wait, since future solutions may make these disappear. In any other case, there can be no solution, so we fail with the (prune-fail) rule. For example, $\forall x, y . \alpha x \equiv y$ has no closed solution for $\alpha$, since $\alpha$ may appear in contexts where $y$ is not bound.

We present only the rules for when pruning fails, since the rules where it succeeds are simplification rules. A complete account of pruning is given by Abel and Pientka [15].

\section{Type-checks}

After a substitution has been applied, a problem $\forall \Gamma . s: S \equiv t: T$ may be solved, that is, $s=t$, and the (reflexivity) rule can be applied. However, to consider the problem solved, $S$ and $T$ must also be equal, and the solution must type-check against $T$. If this succeeds, the problem can be removed from the metacontext. If this fails, then the problem is stuck. Note that the typechecking here occurs relative to the declarative system: we do not generate further constraints when type-checking here. Likewise, if a problem has no metavariables but its two sides are not equal, the metacontext has no solution.

Our rewrite rules use these type-checks as guards when adding solutions or deleting reflexive equations, so it becomes stuck when failures are encountered. In Section 6.4 we show how to proceed despite these failures.

\subsection{A worked-through example}

To give a concrete example of the above algorithm, we apply it to the constraints generated when type-checking the code from Section 2.1.1. Suppose that, when typechecking, $\beta_{1}, \beta_{2}, \beta_{3}$ are the names given to the respective values omitted as .

After type-checking (and applying trivial equalities), the metacontext $\Delta=\left\{P_{1}, P_{2}, P_{3}, P_{4}, P_{5}, P_{6}, P_{7}\right\}$ is generated, where the problems $P_{1} \ldots P_{7}$ are defined in Figure 5 .

The unification algorithm proceeds as follows:

1. We apply (rigid-pi) to the metacontext, which decomposes $P_{1}$ into $\alpha_{1} \equiv$ Set and $\alpha_{2} \equiv \lambda a \ldots .(b n)$. We can apply (inversion) with an empty spine twice to solve the (trivial) equations. Our metacontext is now $\left\{\alpha_{1}:=\right.$ Set, $\left.\alpha_{2}:=(\lambda a \ldots(b n)), P_{2}, \ldots, P_{7}\right\}$.

2. The solutions from $P_{1}$ can be substituted forwards in the metacontext (using the omitted simplification rules), transforming the left-hand side of $P_{2}$ into :

$$
\begin{array}{r}
\Pi(\mathrm{Nat} \rightarrow \mathrm{Set}) \lambda b . \\
\Pi(\mathrm{Nat}) \lambda n . \\
\Pi\left(\Pi \text { Nat } \lambda i . \beta_{1} \rightarrow b i \rightarrow b(\operatorname{Succ} i)\right) \lambda_{-} . \\
\Pi(b 0) \lambda_{-} . \\
\Pi\left(\operatorname{Vec} \beta_{1} n\right) \lambda_{-} .
\end{array}
$$

3. We can repeat the above two steps for $P_{2}$ and $P_{3}$ to obtain $\alpha_{3}:=$ Nat $\rightarrow$ Set, $\alpha_{4}:=(\lambda b \ldots(b n)), \alpha_{5}:=$ Nat, and

$$
\alpha_{6}:=\quad \lambda n . \Pi
$$

( $\Pi$ Nat $\left.\lambda i . \Pi \beta_{1} \lambda_{-} . \Pi \beta_{2} i \lambda_{-} . \beta_{2}(\operatorname{Succ} i)\right)$

$$
\begin{array}{r}
\lambda_{-} \Pi\left(\beta_{2} 0\right) \lambda_{-} . \\
\Pi\left(\operatorname{Vec} \beta_{1} n\right) \lambda_{-} . \\
\left(\beta_{2} n\right)
\end{array}
$$

4. After substituting our solutions in $P_{4}$, and once again applying (rigid-pi), we finally obtain interesting equalities:

$$
\begin{array}{r}
\left(\Pi \text { Nat } \lambda i . \Pi \beta_{1} \lambda_{-} . \Pi \beta_{2} i \lambda_{-} \cdot \beta_{2}(\operatorname{Succ} i)\right) \equiv \\
\left(\Pi \text { Nat } \lambda i . \Pi \text { Nat } \lambda_{-} .\right. \\
\left.\Pi(\operatorname{Vec} \text { Nat } i) \lambda_{-} .(\operatorname{Vec} \operatorname{Nat}(\operatorname{Succ} i))\right)
\end{array}
$$

and

$$
\lambda_{-} . \Pi(b 0) \lambda_{-} . \Pi\left(\operatorname{Vec} \beta_{1} \beta_{3}\right) \lambda_{-} \cdot \beta_{2} \beta_{3} \equiv \alpha_{7}
$$

The latter equation is solved trivially, but the first requires further decomposition. Again, we can apply (rigid-pi), to obtain Nat $\equiv$ Nat, which we can delete with (reflexivity), and (after using a simplification rule to $\eta$-expand):

$$
\begin{array}{r}
\left.\forall i . \quad \Pi \beta_{1} \lambda_{-} \cdot \Pi \beta_{2} i \lambda_{-} \cdot \beta_{2}(\operatorname{Succ} i)\right) \equiv \\
\left.\Pi \text { Nat } \lambda_{-} . \Pi(\operatorname{Vec} N a t i) \lambda_{-} .(\operatorname{Vec} \operatorname{Nat}(\operatorname{Succ} i))\right)
\end{array}
$$

We can then apply (rigid-pi) to obtain $\beta_{1}:=$ Nat, the solution to the first underscore, along with (etaexpanded)

$$
\forall i . \quad \begin{array}{r}
\left.\Pi \beta_{2} i \lambda_{-} \cdot \beta_{2}(\operatorname{Succ} i)\right) \\
\left.\Pi(\operatorname{Vec} N a t i) \lambda_{-} .(\operatorname{Vec} N a t(\operatorname{Succ} i))\right)
\end{array}
$$

Applying (rigid-pi) here now gives us two higher-order problems: 


\begin{tabular}{|c|c|c|}
\hline$P_{1}:$ & $\Pi \alpha_{1} \alpha_{2} \equiv$ & $\begin{array}{r}\Pi \text { Set } \lambda a . \\
\Pi(\text { Nat } \rightarrow \text { Set }) \lambda b . \\
\Pi(\text { Nat }) \lambda n . \\
\Pi\left(\Pi \text { Nat } \lambda i . \Pi a \lambda_{-} . \Pi(b i) \lambda_{-}(b(\operatorname{Succ} i))\right) \lambda_{-} . \\
\Pi(b 0) \lambda_{-} . \\
\Pi(\operatorname{Vec} a n) \lambda_{-} \\
(b n) \\
\text { (vecFoldr has a function type })\end{array}$ \\
\hline$P_{2}:$ & $\alpha_{2} \beta_{1} \equiv$ & $\begin{array}{r}\Pi \alpha_{3} \alpha_{4} \\
\text { (vecFoldr } \beta_{1} \text { has a function type) }\end{array}$ \\
\hline$P_{3}:$ & $\alpha_{4} \beta_{2} \equiv$ & $\begin{array}{r}\Pi \alpha_{5} \alpha_{6} \\
\text { (vecFoldr } \beta_{1} \beta_{2} \text { has a function type) }\end{array}$ \\
\hline$P_{4}:$ & \multicolumn{2}{|c|}{$\begin{array}{c}\left.\alpha_{6} \beta_{3} \equiv \quad M\left(\Pi \text { Nat } \lambda i . \Pi \text { Nat } \lambda_{-} . \Pi(\operatorname{Vec} \text { Nat } i) \lambda_{-} .(\text {Vec Nat (Succ } i)\right)\right) \alpha_{7} \\
\text { (vecFoldr } \beta_{1} \beta_{2} \beta_{3} \text { has a function type whose domain matches type of doubleHead) }\end{array}$} \\
\hline$P_{5}:$ & $\begin{array}{l}\alpha_{7} \text { doubleHead } \equiv \\
\quad(\operatorname{vecFoldr} . .\end{array}$ & $\begin{array}{r}\Pi(\text { Vec Nat } 0) \alpha_{8} \\
\text { has a function type whose domain matches type of Nil Nat) }\end{array}$ \\
\hline$P_{6}:$ & $\begin{array}{l}\alpha_{8}(\text { Nil Nat }) \equiv \\
\quad(\text { vecFold }\end{array}$ & $\begin{array}{r}\Pi\left(\operatorname{Vec} \text { Bool 1) } \alpha_{9}\right. \\
\text { has a function type whose domain matches types of myList) }\end{array}$ \\
\hline$P_{7}:$ & $\alpha_{9}$ myList $\equiv$ & $\begin{array}{r}(\text { Vec Nat 1) } \\
\text { (The return type of the entire application is Vec Nat 1) }\end{array}$ \\
\hline
\end{tabular}

Figure 5: Metacontext from type-checking code of Section 2.1.1.

$$
\begin{aligned}
\forall i . & \beta_{2} i & \equiv(\text { Vec Nat } i) \\
\forall i . & \beta_{2}(\text { Succ } i) & \equiv(\text { Vec Nat }(\text { Succ } i))
\end{aligned}
$$

However, both of these fall neatly into the pattern fragment, so we can apply (inversion) with a spine $i$ to get $\beta_{2}:=\lambda i$. (Vec Nat $\left.i\right)$. Applying this substitution allows us to eliminate the second equation with (reflexivity).

5. Our new solutions for $\beta_{1}, \alpha_{7}$ and $\beta_{2}$ transform $P_{5}$ into

$$
\begin{array}{r}
\Pi(\operatorname{Vec} \text { Nat } 0) \lambda_{-} . \Pi\left(\operatorname{Vec} N a t \beta_{3}\right) \lambda_{-} \cdot \operatorname{Vec} \text { Nat } \beta_{3} \equiv \\
\Pi(\text { Vec Nat } 0) \alpha_{8}
\end{array}
$$

We can solve this similarly as with $P_{1}$, giving a reflexive equality and $\alpha_{8}:=\lambda_{-}$. $\Pi\left(\operatorname{Vec} \operatorname{Nat} \beta_{3}\right) \lambda_{-}$. Vec Nat $\beta_{3}$

6. On $P_{6}$, we get stuck (due to the type error in the code). Applying all our solutions thus far, $P_{6}$ becomes $\Pi\left(\right.$ Vec Nat $\left.\beta_{3}\right) \equiv \Pi\left(\operatorname{Vec}\right.$ Bool 1) $\alpha_{9}$. Repeated rigid decomposition gives Nat $\equiv$ Bool and $\beta_{3} \equiv 1$, but the first equation cannot be simplified, and unification fails.

\section{Replay graphs for message generation}

Here, we describe one approach for dependently-typed error message generation. Specifically, we follow the approach of the Helium Haskell Compiler [2-4], where a unification problem is represented as a graph, with edges representing equality. This format naturally captures the transitive nature of equality using graph reachability. More importantly, it represents the entire unification problem in a global, non-linear way. Thus, instead of iteratively solving a problem and reporting an error as soon as it is encountered, a set of heuristics can be applied to diagnose the problem. These heuristics can see the entire graph, and thus the context surrounding an error. Additionally, they can try modifications on the graph, seeing which make it consistent, and use this information to generate repair hints.

Many heuristics of Helium can be adapted to dependent-types with little modification. However, He- 
lium's graph representation is too simple for dependenttypes, and does not naturally capture the dynamic aspects of higher-order unification.

In this section, we present our solution to this problem. We give a modified unification algorithm that generates a replay graph, representing the steps unification took. Instead of generating solutions from the graph, we perform unification as we normally would, but if an error is encountered, we refer to the graph, applying heuristics to generate a message.

Additionally, since replay graphs capture any failures during unification, they allow us to make our algorithm error-tolerant. When a failure is encountered, we can safely proceed as if it had not happened, since the failure is recorded in the replay graph. We formalize this process in Section 6.4.

\subsection{Constraint graphs for first-order unification}

We begin by explaining first-order unification, since our higher-order replay graphs are easiest to understand in relation to the constraint graphs that systems such as Helium use for unification.

First, consider a set of equality constraints over metavariables and atomic types. To represent such a program graphically, we have nodes in our graph for each atomic type or metavariable. For each constraint $T_{1} \equiv T_{2}$, we add an undirected edge between the nodes for $T_{1}$ and $T_{2}$. Since reachability and equality are both transitive, we can identify the solution for a metavariable as the atomic type in its connected component. If two distinct atomic types are in the same connected component, then no solution to the constraint set exists.

To account for types involving type constructors, such as $\rightarrow$ or List, we use directed structure edges. First, we must establish how to construct a node for a complex type $T$. If $T$ is $\chi S_{1} \ldots S_{n}$ for some type constructor $X$ and types $S_{1} \ldots S_{n}$, then we add a constructor application node to our graph for $T$, an atomic constructor node for $\chi$, and the nodes for each $S_{i}$. The node for each $S_{i}$ is either its atomic type or metavariable node, or its constructor application node if it is a complex type. To reflect the structure of $T$, we add a directed edge from $T$ to $\chi$ labeled ( $\downarrow$, HEAD), and a directed edge from $\chi$ to $T$ labeled ( $\uparrow$, HEAD). Similarly, we add an edge $\left(\downarrow, i_{\chi}\right)$ from $T$ to $S_{i}$ and $\left(\uparrow, i_{\chi}\right)$ from $S_{i}$ to $T$.

For instance, the type $T=T_{1} \rightarrow T_{2}$ would be represented as the following graph:

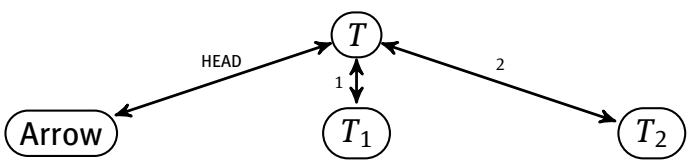

Specifically, the following edges would be generated:

$$
\begin{gathered}
(T, \rightarrow, \downarrow, \mathrm{HEAD}),(\rightarrow, T, \uparrow, \mathrm{HEAD}), \\
\left(T, T_{1}, \downarrow, 1_{\text {Arrow }}\right),\left(T_{1}, T, \uparrow, 1_{\text {Arrow }}\right), \\
\left(T, T_{2}, \downarrow, 2_{\text {Arrow }}\right),\left(T_{2}, T, \uparrow, 2_{\text {Arrow }}\right)
\end{gathered}
$$

As an intuition behind this notation, we imagine the syntax tree for a term with the root at the top. The edges labeled $\downarrow$ represent moves down, from the root towards the leaves, whereas edges labeled $\uparrow$ are moves from the leaves towards the root.

With a notion of how to represent complex terms as graphs, the final detail is how to represent equalities between complex nodes. We can form paths between nodes using both directed and undirected edges, but this includes too many paths. In our previous example, if we have $S_{1} \rightarrow T_{1}$ connected to $S_{2} \rightarrow T_{2}$, we do not want a path between $S_{1}$ and $T_{2}$, since that equality is not implied. Instead, we must consider which paths are equality paths.

Definition 2. The set of equality paths is defined inductively:

- Any undirected path is an equality path.

- A path is also an equality path if it has the form

$$
{\overline{U_{i}}}^{i},(S, T, \uparrow, L), P,(T, S, \downarrow, L),{\overline{U_{j}}}^{j}
$$

where $P$ is a smaller equality path, ${\overline{U_{i}}}^{i}$ and ${\overline{U_{j}}}_{j}^{j}$ are (possibly empty) undirected paths, $S$ and $T$ are nodes, and $L \in\left\{\operatorname{HEAD}, 1_{\chi}, 2_{\chi}, \ldots\right\}$.

Essentially, moves along directed edges must be well nested, with $\downarrow$ preceding $\uparrow$. This corresponds to our equality rules for constructors: if two terms are equal, then they must have equal constructors and equal arguments. If an equality path connects two non-equal nodes for atomic types or type constructors, then it is called an error-path. Labelling the argument nodes with the constructor $\chi$ ensures that we do not have redundant equality paths between arguments of terms with different heads, since there is already an error path between the heads.

An alternate, but equivalent, way to think of an equality path is by implicit edges. Whenever there is a path between two complex terms, there are implicit undirected edges between their heads and corresponding arguments.

A set of unification constraints has a solution if and only if two conditions hold. First, the graph for the constraint set must not contain any error paths. Secondly, no 
metavariable can have a path to itself through a directed edge, since this prohibits finite solutions. The second condition is essentially an occurs-check.

The advantage of this approach is that the graph provides a single representation of the entire unification problem. The various equalities can be considered simultaneously by heuristics, diagnosing the type error by selecting a set of undirected edges whose removal will eliminate all error paths. The program points that induced the constraints for these edges are then presented as the cause of the type error. Edges can be added or removed, so that heuristics can search for specific changes that will repair the graph. Moreover, the representation is unbiased: the order in which constraints are emitted has no effect on the final graph.

\section{A first-order example}

Consider checking the Haskell program:

$(++)[1,2,3]$ [True] i.e. concatenating two lists using prefix notation. The function ++ has type $[a]->$ [a] $\rightarrow$ [a], so when type-checking the code, $a$ is instantiated with a fresh unification variable $\beta$, and we get the following constraints:

$$
\begin{array}{rc}
\text { List } \beta \rightarrow \text { List } \beta \rightarrow \text { List } \beta \equiv & \begin{array}{c}
\alpha_{1} \rightarrow \alpha_{2} \\
((++) \text { is a function })
\end{array} \\
\alpha_{1} \equiv & \text { List Int }
\end{array}
$$

(argument $[1,2,3]$ matches function domain type)

$$
\begin{array}{cc}
\alpha_{2} \equiv & \alpha_{3} \rightarrow \alpha_{4} \\
((++) & [1,2,3] \text { is a function }) \\
\alpha_{3} \equiv & \text { List Bool }
\end{array}
$$

(argument [False] matches function domain type)

In Helium, this would be represented as the constraint graph shown in Figure 6. The following error path between Int and Bool is present:

(Int, List Int, $\left.\uparrow, 1_{\text {List }}\right)$, (List Int, $\left.\alpha_{1}\right),\left(\alpha_{1}, \alpha_{1} \rightarrow \alpha_{2}, \uparrow, 1_{\text {Arrow }}\right)$, $\left(\alpha_{1} \rightarrow \alpha_{2}\right.$, List $\beta \rightarrow$ List $\beta \rightarrow$ List $\beta$ ), (List $\beta \rightarrow$ List $\beta \rightarrow$ List $\beta$, List $\beta, \downarrow, 1_{\text {Arrow }}$ ), (List $\left.\beta, \beta, \downarrow, 1_{\text {List }}\right),\left(\beta\right.$, List $\left.\beta, \uparrow, 1_{\text {List }}\right)$, (List $\beta$, List $\beta \rightarrow$ List $\beta, \uparrow, 1_{\text {Arrow }}$ ), (List $\beta \rightarrow$ List $\beta$, List $\beta \rightarrow$ List $\beta \rightarrow$ List $\beta$, List $\beta, \uparrow, 2_{\text {Arrow }}$ ),

(List $\beta \rightarrow$ List $\beta \rightarrow$ List $\beta, \alpha_{1} \rightarrow \alpha_{2}$ ), $\left(\alpha_{1} \rightarrow \alpha_{2}, \alpha_{2}, \downarrow, 2_{\text {Arrow }}\right),\left(\alpha_{2}, \alpha_{3} \rightarrow \alpha_{4}\right)$, $\left(\alpha_{3} \rightarrow \alpha_{4}, \alpha_{3}, \downarrow, 1_{\text {Arrow }}\right),\left(\alpha_{3}\right.$, List Bool), (List Bool, Bool, $\downarrow, 1_{\text {List }}$ )
Notice the nesting structure: to reach $\beta$, we go $\uparrow 1_{\text {List }}, \uparrow 1_{\text {Arrow }}, \downarrow 1_{\text {Arrow }}, \downarrow 1_{\text {List }}$, and to leave $\beta$ we go $\uparrow 1_{\text {List }}, \uparrow 2_{\text {Arrow }}, \uparrow 1_{\text {Arrow }}, \downarrow 1_{\text {Arrow }}, \downarrow 2_{\text {Arrow }}, \downarrow 1_{\text {List }}$.

The intuition behind this path is as follow: the injectivity of constructors means that if List $\beta \rightarrow$ List $\beta \rightarrow$ List $\beta \equiv \alpha_{1} \rightarrow \alpha_{2}$, then there are implied equalities (i.e. implied edges) List $\beta \equiv \alpha_{1}$ and List $\beta \rightarrow$ List $\beta \equiv \alpha_{2}$. Since $\alpha_{2} \equiv \alpha_{3} \rightarrow \alpha_{4}$, there are implied equalities List $\beta \equiv \alpha_{3}$ and List $\beta \equiv \alpha_{4}$. Since there's an edge $\alpha_{1} \equiv$ List Int and $\alpha_{3} \equiv$ List Bool, there's a path List Int $\equiv$ List Bool through $\alpha_{1} \equiv$ List $\beta \equiv \alpha_{3}$. Finally, this implies the equality Int $\equiv$ Bool.

\subsection{Replay graphs for higher-order unification}

In this section, we introduce a graph representation of higher-order unification problem sets, which we call replay graphs. We describe methods for generating them from constraints, as well as for analyzing them in order to diagnose the likely cause of errors.

The first-order approach of Section 6.1 fails in a higherorder setting. Since we are performing unification on both types and terms, we may have to unify terms involving applications of non-injective functions to types and type indices. The equality paths of first-order unification are correct only because of the injectivity of constructors. For example, the following equation holds:

$$
(\lambda x . \lambda y . \text { Zero) Zero Zero } \equiv(\lambda x . \lambda y . x) \text { Zero (Succ Zero) }
$$

However, we cannot conclude from this that $(\lambda x . \lambda y$. Zero $) \equiv(\lambda x . \lambda y . x)$, or that Succ Zero $\equiv$ Zero.

Likewise, the simplification rules of our unification algorithm transform problems so that solutions can be found. For example, $\eta$-expansion transforms unification of functions into unification of their bodies and decomposes constraints on dependent pairs to constraints on their components. Similarly, when a solution for a metavariable is found, the new value is substituted into terms of other problems, which are then evaluated. Such operations do not have an obvious graph-based counterpart.

To represent a higher-order problem graphically, we decompose it into a number of first-order equalities by running higher-order unification and recording the subproblems and solutions that are generated. When an error is found and a message needs to be generated, the replay graph is analyzed to diagnose the cause of the error. Thus, the graph can be ignored when finding solutions for metavariables, but it can be a rich source of information 


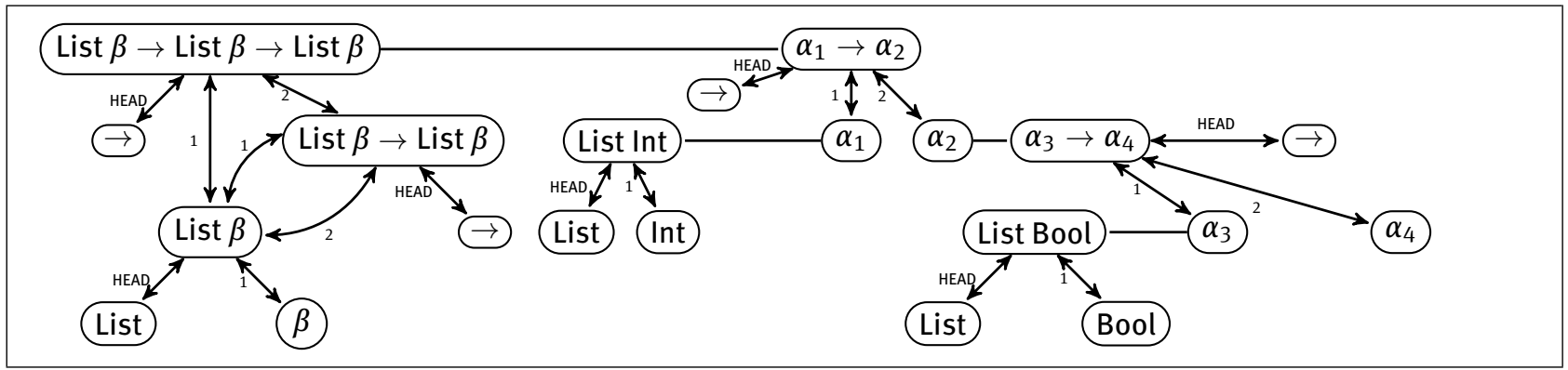

Figure 6: First-order type graph from checking $(++)[1,2,3]$ [False ].

about unifications that have taken place whenever an error message needs to be generated.

Because replay graphs rely on our unmodified unification algorithm, they can exhibit some bias, as the order in which unification problems are solved can still somewhat affect the error message output. Unlike with first-order problems, we cannot solve the problems in any order, since problems outside of the pattern fragment can never be solved until other solutions push them into the pattern fragment. Such restrictions are inherent to dynamic unification with dependent-types, but we propose counterfactual unification (Section 7) as a theoretical tool to explore all orderings which do not cause unification to become stuck.

\subsubsection{Graph structure}

Our graph structure is very similar to the first-order version, though enriched to allow for a unified type-term language. At its core, our graph consists of a set of nodes, which represent terms, and a set of edges between those nodes, representing structural dependencies and equalities between nodes. The specification for nodes and edges is given in Figure 7(a).

\section{Nodes}

Our nodes come in four varieties. There is a single node for each metavariable. Nodes for rigid heads are also present, representing the injective constructors of our language, such as Set, $\Pi$ or $\Sigma$. Additionally, a rigid head may be a variable, since we cannot generally assume $x \equiv y$ for free variables $x, y$ in a term. Constructor application nodes represent simple terms built from constructors, like $\Pi S T$.

To account for the remaining, more complex terms, such as lambdas or neutral terms, we have raw term nodes, which simply store an entire term in a single node. These act as placeholders, storing the term so we can perform substitution and evaluation on it as we find solutions for metavariables. They then provide a connection between a term and its evaluation.

In our implementation, we allow for multiple nodes representing the same term to be in our vertex set. This ensures that unrelated program segments do not conflate their errors. For instance, if there are two unrelated errors involving the Set constructor, having a path between them through a single Set constructor could result in a diagnosis completely unrelated to the actual cause of the error.

\section{Edges}

Our edges come in the same two varieties as first-order graphs. Undirected equality edges are written $[u, v]$. These denote that the terms defined by two nodes should be definitionally equal. Directed structure edges are written ( $u, v, d, L)$, where $D \in\{\uparrow, \downarrow\}$, and $L$ is either HEAD or $i_{r}$ for some rigid head $r$, denoting the children corresponding to the rigid head and $i$ th arguments of a complex term. For example, the node for $\Pi S T$ is an application node, with $\downarrow$ edges to $\Pi, S$ and $T$, labeled HEAD, $1_{\Pi}, 2_{\Pi}$ respectively, and corresponding $\uparrow$ edges in the opposite direction.

\subsubsection{Constructing replay graphs}

As is standard, a graph $\mathcal{G}$ is simply a pair consisting of a set of vertices, and a set of edges over those vertices. The graph union $\mathcal{G}_{1} \cup \mathcal{G}_{2}$ is simply the union of its vertex and edge sets.

Overall, we wish to represent each equality encountered during unification in our graph. We add equality edges in three cases: between the two terms of a unification problem, between a metavariable and its solution, and between a term and its evaluated form after applying previous solutions. 


\section{Vertices

$\begin{array}{rrr}\mathcal{V}::= & \alpha & \text { (Metavariable Node) } \\ \mid & r & \text { (Rigid Head) } \\ \mid & \operatorname{App}_{t} & \text { (Rigid Head Application) } \\ \mid & \operatorname{Raw}(t) & \text { (Raw Term node) }\end{array}$

\section{Rigid Heads}

$$
r::=\text { Set }|\Pi| \Sigma \mid \text { funElim }\left|\pi_{1}\right| \pi_{2} \mid \chi
$$

Edges

$$
\begin{array}{lrr}
D::= & \uparrow \mid \downarrow & \\
L::= & \text { HEAD } \mid i_{r} \quad i \in \mathbb{N} & \\
\mathcal{E}::= & \left(\mathcal{V}_{1}, \mathcal{V}_{2}, D, L\right) & \text { (Applied term) } \\
\mid & {\left[\mathcal{V}_{1}, \mathcal{V}_{2}\right]} & \text { (Equality Edge) }
\end{array}
$$

\section{$V(t)=V$ Term Vertices}

$$
\begin{aligned}
\mathcal{V}(t) & =t \quad \text { if } t \text { is a rigid head } \\
\mathcal{V}(t) & =\mathcal{V}\left(t^{\prime}\right) \quad \text { if } t \rightarrow^{\star} t^{\prime} \\
\mathcal{V}(\alpha) & =\alpha \\
\mathcal{V}(\Pi S T) & =\operatorname{App}_{\Pi s T} \\
\mathcal{V}(\Sigma S T) & =\operatorname{App}_{\Sigma S T} \\
\mathcal{V}(\text { funElim } t) & =\operatorname{App}_{\text {funElim } t} \\
\mathcal{V}\left(\alpha{\overline{e_{i}}}^{i}\right) & =\operatorname{Raw}\left(\alpha{\overline{e_{i}}}^{i}\right) \\
\mathcal{V}(\lambda x . t) & =\operatorname{Raw}(\lambda x . t) \\
\mathcal{V}((s, t)) & =\operatorname{Raw}((s, t))
\end{aligned}
$$

(b) Term vertices.

(a) Replay graphs: syntax.

\section{$\varepsilon(t)=\bar{\varepsilon}_{i}^{i}$ Term Edges}

$$
\begin{aligned}
& \varepsilon(t)= \\
& \varepsilon(\Pi S T)= \\
& \mathcal{E}(\Sigma S T)= \\
& \mathcal{E}(\text { funElim } t)= \\
& \left(\operatorname{App}_{\Pi S T}, \Pi, \downarrow, \mathrm{HEAD}\right),\left(\operatorname{App}_{\Pi S T}, S, \downarrow, 1\right),\left(\operatorname{App}_{\Pi S T}, T, \downarrow, 2\right) \\
& \left(\Pi, \operatorname{App}_{\Pi S T}, \uparrow, \mathrm{HEAD}\right),\left(S, \operatorname{App}_{\Pi S T}, \uparrow, 1\right),\left(T, \operatorname{App}_{\Pi S T}, \uparrow, 2\right) \\
& \left(\operatorname{App}_{\Sigma S T}, \Sigma, \downarrow, \mathrm{HEAD}\right),\left(\operatorname{App}_{\Sigma S T}, S, \downarrow, 1\right),\left(\operatorname{App}_{\Sigma S T}, T, \downarrow, 2\right) \\
& \text { ( } \left.\Sigma, \operatorname{App}_{\Sigma S T}, \uparrow, \mathrm{HEAD}\right),\left(S, \operatorname{App}_{\Sigma S T}, \uparrow, 1\right),\left(T, \operatorname{App}_{\Sigma S T}, \uparrow, 2\right) \\
& \left(\mathrm{App}_{\text {funElim } t} \text {, funElim, } \mathrm{L}\right),\left(\mathrm{App}_{\text {funElim } t}, \mathcal{V}(t), \mathrm{R}\right)
\end{aligned}
$$

$\mathcal{E}_{\mathcal{G}}(\Delta)={\overline{\varepsilon_{i}}}^{i}$ Metacontext Edges

$$
\begin{aligned}
\mathcal{E}_{\mathcal{G}}(\cdot)= & \\
\mathcal{E}_{\mathcal{G}}(\Delta, \alpha: T)= & \\
\mathcal{E}_{\mathcal{G}}(\Delta, \alpha:=t: T)= & \mathcal{E}_{\mathcal{G}}(\Delta) \\
\mathcal{E}_{\mathcal{G}}(\Delta,(s: S) \equiv(t: T))= & \mathcal{E}_{\mathcal{G}}(\Delta), \mathcal{E}(\operatorname{subTms}(t)),[\mathcal{V}(\alpha), \mathcal{V}(t)] \\
\mathcal{E}_{\mathcal{G}}(\Delta, \forall \Gamma . P)= & \mathcal{E}_{\mathcal{G}}(\Delta), \mathcal{E}(\operatorname{subTms}(s, t)),[\mathcal{V}(s), \mathcal{V}(t)] \\
& \mathcal{E}_{\mathcal{G}}(\Delta), \mathcal{E}_{\mathcal{G}}\left(\left[\Gamma \Leftrightarrow \Gamma^{\prime}\right] P\right) \quad \text { where } \Gamma^{\prime} \cap \mathrm{FV}(\mathcal{G})=\emptyset
\end{aligned}
$$

\begin{tabular}{l|l}
$\mathcal{G}_{\text {eval }}(\mathcal{G}, \Delta)=\mathcal{G}^{\prime}$ & Evaluation Edges
\end{tabular}

$$
\mathcal{G}_{\text {eval }}(\mathcal{G}, \Delta)=\quad \mathcal{G} \cup\{[\mathcal{V}(s), \mathcal{V}([\alpha \boxminus t] s)] \mid \alpha:=t: T \in \Delta, \operatorname{Raw}(s) \in \mathcal{G}, \alpha \in \operatorname{FMV}(s)\}
$$

(c) Graph construction rules: edges.

Figure 7: Replay graph construction. 
The formal rules for constructing our graph are given in Figure 7. We gradually build the graphs for a unification run. First, we have the vertex $\mathcal{V}(t)$ for each subterm $t$, given in Figure 7(b). Raw terms, rigid heads, and metavariables have their own nodes, while terms with constructor heads are given application nodes. Note that we are flexible with our syntax, also assigning nodes to eliminators.

The rules for generating edges are given in Figure 7(c). To build the complete graph for term $t$, we compute its vertex, and add the edges, denoted $\mathcal{E}(t)$, that represent the structure for the term. This set is empty for metavariables, rigid heads and raw terms, but contains the HEAD and numbered argument edges for the complex term nodes described in Section 6.1.

The graph for a metacontext $\Delta$ contains $\mathcal{V}(t)$ and $\varepsilon(t)$ for each sub-term $t$ of $\Delta$. Additionally, it contains undirected equality edges for each equality and solution assignment contained in $\Delta$. We denote this graph as $\mathcal{E}_{\mathcal{G}}(\Delta)$, referencing the graph $\mathcal{G}$ constructed so far so that we can generate fresh variables.

When adding a constraint of the form $\forall \Gamma$. $C$ to our graph, we replace each variable in $\Gamma$ with a free, unconstrained variable, ensuring that the constraints are indeed satisfied for any possible $\Gamma$.

Finally, we have rules to construct the evaluation edges for a metacontext $\Delta$. These account for the fact that checking of dependent-types must perform evaluation of terms. When we have a solution $\alpha:=t$ in $\Delta$, and $\alpha$ occurs in some term $s$ in $\Delta$, we add an edge from $s$ in its original raw form to $s$ after substituting $t$ for $\alpha$ and evaluating. These rules are crucial for adapting type graphs to dependent-types, and make up for Helium's lack of support for type-level computation.

The connected components (via equality paths) of our graph are called equivalence groups: any two terms in an equivalence group should be equal after the appropriate substitutions for metavariables are made.

\section{Example: metacontext graphs}

Figure 8(a) shows the graph for the metacontext:

$$
\Delta=\alpha: \text { Set, } ? \alpha \equiv \mathrm{Eq} 00, ? \alpha \equiv \mathrm{Eq}(\text { Succ } 0) 0
$$

(While the inductive types Eq and constructors 0, Succ are not part of our calculus, we use them to provide more realistic examples.) The nodes consist of rigid heads for 0 , Succ, and Eq, a metavariable node $\alpha$, and constructor application nodes for Succ 0, Eq 00 and Eq (Succ 0) 0.

For term edges, we have directed edges from Eq 00 to nodes for Eq, 0 and 0 , labeled HEAD, $1_{\mathrm{Eq}}$ and $2_{\mathrm{Eq}}$ respec-

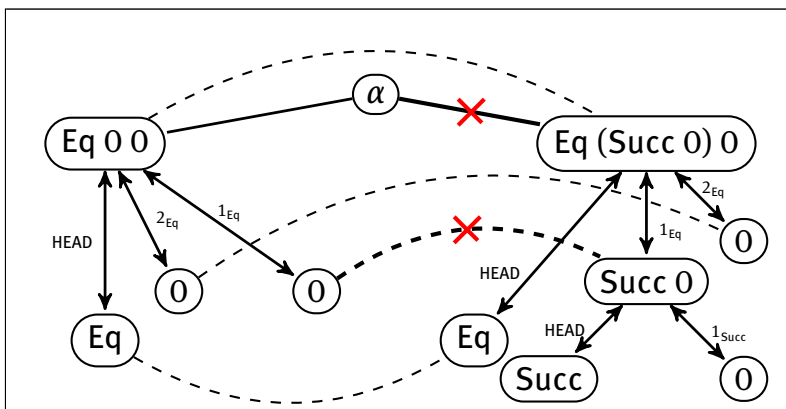

(a) Replay graph for $\{\alpha \equiv \mathrm{Eq} 00, \alpha \equiv \mathrm{Eq}$ (Succ 0 ) 0$\}$. Dashed edges are implied by equality paths, and the crossed-out edges are a possible diagnosis.

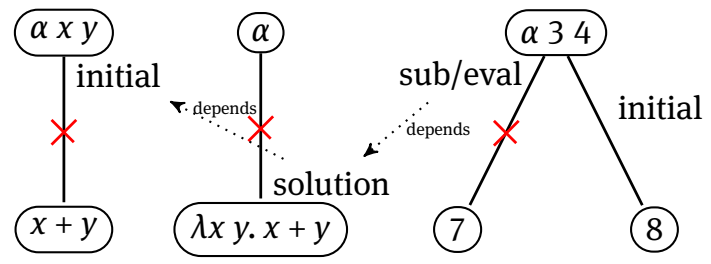

(b) Replay graph for $\{\forall x, y . \alpha x y \equiv x+y, \alpha 34 \equiv 8\}$. Inversion gives $\alpha:=\lambda x$. $x+y$, so $\alpha 34$ gets an evaluation edge to 7. The crossed-out edges are a possible diagnosis.

Figure 8: Example replay graphs.

tively, with similar edges in place for Eq (Succ 0) 0 and Succ 0.

Finally, we have the equality edges between $\alpha$ and the two Eq terms. These edges, combined with the directed edges, induce the following equality paths:

$$
\begin{aligned}
& \text { Eq, ( } \uparrow, \text { HEAD), Eq } 00, \quad \alpha, \quad \text { Eq (Succ 0) } 0,(\downarrow, \text { HEAD), Eq } \\
& 0,\left(\uparrow, 1_{\mathrm{Eq}}\right), \mathrm{Eq} 00, \quad \alpha, \quad \mathrm{Eq}(\operatorname{Succ} 0) 0,\left(\downarrow, 1_{\mathrm{Eq}}\right) \text {, Succ } 0 \\
& \left.0,\left(\uparrow, 2_{\mathrm{Eq}}\right) \text {, Eq } 00, \quad \alpha, \quad \text { Eq (Succ } 0\right) 0,\left(\downarrow, 2_{\mathrm{Eq}}\right), 0
\end{aligned}
$$

There is an error path between 0 and Succ 0 . One possible diagnosis, denoted by the crossed out edges, is to delete the edge between $\alpha$ and Eq (Succ 0) 0 , thus deleting the equality path between 0 and Succ 0 . However, deleting the edge between Eq 00 and $\alpha$ is an equally valid diagnosis, and heuristics must be used to determine the one that is the likely error cause.

\section{Constructing the complete graph}

Given the rules for constructing a graph for a metacontext, we can construct the replay graph for an entire unification run.

Suppose the unmodified unification algorithm, when given initial metacontext $\Delta_{1}$, rewrites it as the sequence 
$\Delta_{1} \mapsto \Delta_{2} \mapsto \ldots \mapsto \Delta_{n}$, where $\Delta_{n}$ is final. Then we can define a series of graphs for each step as follows:

$$
\begin{array}{lr}
\mathcal{G}_{0}= & \emptyset, \\
\mathcal{G}_{i}= & \mathcal{G}_{\text {eval }}\left(\mathcal{G}_{i-1} \cup \mathcal{E}\left(\mathcal{G}_{i-1}, \Delta_{i}\right)\right)
\end{array}
$$

That is, we build our final graph adding the edges from each intermediate metacontext $\Delta_{i}$, then adding the edges between terms and their evaluated forms after applying solutions from $\Delta_{i}$.

We say that $\mathcal{G}_{n}$ is the replay graph of unification of $\Delta_{1}$. The graph begins empty, and then has nodes and structure edges for all terms in $\Delta_{1}$ added. Each time a new solution is generated, or a problem is decomposed by the unification algorithm, the corresponding edges are generated, and will be included in the final graph. This provides us with a global overview of the constraints induced by the initial metacontext, allowing us to use heuristics to diagnose errors and recommend repairs.

\subsubsection{Edge dependencies}

In a practical implementation, to facilitate message generation, we can keep a dependency tree of generated constraints. The sub-constraints of a rigid-rigid unification or $\eta$-expansion $P$ are dependent on $P$, and the definition $\alpha:=t$ from solving a flex-rigid or flex-flex problem $P$ is dependent on $P$. Updates are dependent on their definitions (which in turn may be dependent on other problems). Thus, when an edge $\varepsilon$ is diagnosed as the source of the error, we can locate the error at the original source-code that induced the constraint.

\section{Example: evaluation edges}

Figure $8(\mathrm{~b})$ shows a replay graph (with directed edges omitted) for the following metacontext $\Delta_{1}$ :

$$
\alpha: \text { Nat } \rightarrow \text { Nat } \rightarrow \text { Nat, } \forall x, y . \alpha x y \equiv x+y, \alpha 34 \equiv 8
$$

Since $\alpha 34 \equiv 8$ is not in the pattern fragment, unification begins by applying inversion on the first problem, yielding $\alpha:=\lambda x y . x+y$. We can then substitute this for $\alpha$ to obtain the problem $3+4=8$, which fails.

In our replay graph, the metacontext graph for $\Delta_{1}$ contains an equality edge between the raw terms $\alpha x y$ and $x+y$, and an equality edge between the raw term $\alpha 34$ and the rigid head 8 . The solution from inversion creates a new metacontext with the edge between the metavariable node $\alpha$ and the raw term $\lambda x y \cdot x+y$. We then use $\mathcal{G}_{\text {eval }}$ to construct the evaluation edge from the raw term $\alpha 34$ to the rigid head 7 .

This example shows how the raw term nodes act as connectors in the graph, connecting the definition from the initial problem set to the results of evaluation.

\section{Example: constraint graph for code (Figure 9)}

We return to the code from Section 2.1.1, describing how a replay graph is constructed from the unification described in Section 5.3. After solving $P_{4}$, we have a path from $\alpha_{8}$ (Nil Nat) to $\Pi$ (Vec Bool 1) $\alpha_{9}$ (i.e. $P_{6}$ after substitutions). From solving $P_{5}$, we get solutions for $\alpha_{8}$ and $\beta_{2}$, which give us an edge from $\alpha_{8}$ (Nil Nat) to $\Pi\left(\beta_{2} \beta_{3}\right) \lambda_{-}$. $\beta_{2} \beta_{3}$, and an edge from $\beta_{2} \beta_{3}$ to Vec Nat $\beta_{3}$.

After these update edges are added, we have an error path between Nat and Bool. (Notice the nesting between travelling up two 1 edges from Nat and down the two 1 edges from Bool).

To generate the error message from Section 2.1.1, we look at the end points of the path, finding the equality edges closest to each endpoint, and getting the source location associated with each edge (by tracing dependencies back to an initial edge). Hence, when identifying locations relevant to the error, we can trace $\left(\beta_{1} \beta_{2}\right.$, Vec Nat $\left.\beta_{3}\right)$ to the constraint generated when type-checking the application vecFoldr _ _ _ doubleHead, and the edge into $\Pi$ (Vec Bool 1) from the type of myList in the context.

\subsection{Error diagnosis and heuristics}

When unification solving is complete, we have a final replay graph $G$. A constraint set is unsatisfiable if two incompatible nodes are connected by an equality path: a sequence of edges where moves up and down directed edges are properly nested (as defined in Section 6.1). A pair of nodes are incompatible if they are distinct rigid heads, or if one is a rigid head and one is a constructor application. Such a path is called an error path.

A diagnosis for an error path is a set of edges which, when removed, also deletes the error path. In order to deal with the density of our graph, we implicitly remove all edges from constraints dependent on $C$ when we remove $C$. This prevents error messages from containing information about intermediate constraints which are not directly found in the initial metacontext, and thus have a nebulous source location. Figure 8 shows two replay graphs, along with a possible diagnosis for each.

There are, of course, many possible diagnoses for an unsatisfiable path. Knowing the correct diagnosis is not 


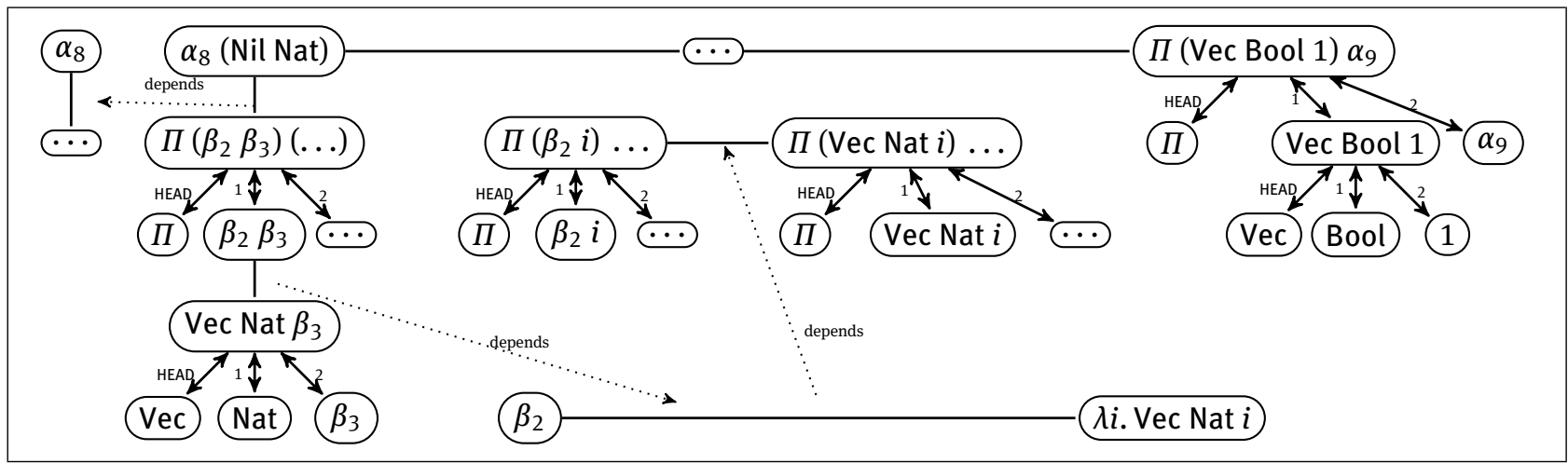

Figure 9: Replay graph for error from Section 2.1.1.

always possible, since in many cases this requires knowing the programmer's intention. As in Helium, we use two sorts of heuristics for deciding which edges to blame. Avoid heuristics mark certain edges unfit for removal. Voting heuristics, conversely, assign votes to each edge. The edge which, after running all heuristics, has the most votes and is not marked unfit, will be removed. This process is repeated until the two conflicting nodes are disconnected.

The heuristics we discuss here form a solid base for diagnosis, but further research is necessary to develop more refined heuristics.

\section{Edge information and inserting messages}

Each edge, when generated, is paired with information about its creation, location in the source code, etc. This information can be accessed by the heuristics, allowing them to use this information when diagnosing the probable cause of the error. The edge information is also accessed during error message generation, so that the printed messages can be as detailed as necessary.

When a heuristic is examining an edge, it can also modify the stored information for that edge. Through this mechanism, we can suggest fixes or provide other information which was obtained during the global analysis of the replay graph, enriching the textual errors presented to the user.

\section{First-order heuristics}

Not all of the Helium heuristics are applicable to dependent-types. We outline here the heuristics from Helium [3], along with some variants, which we included in our implementation.
Participation-ratio: Edges on multiple error paths are more likely to be the cause of an error, so we avoid those on few paths.

First-come first-blamed: When all else is equal, ties are broken arbitrarily.

Permutation: If swapping two arguments to a function can repair the replay graph, the application edge is blamed, and the swap is recommended as a fix.

Application: The expected number of arguments for a function is determined from a replay graph. Too many are given if an argument is given to a value without a $\Pi$ type, and too few when a value is used where a non- $\Pi$ type is expected. Adding or removing arguments is suggested as a possible fix.

\section{Dependently-typed heuristics}

Some of the core Helium heuristics can be adapted to the classes of errors which are specific to dependently-typed languages.

One key difference from simple types is the presence of type indices. While our core calculus has no inductive types, most languages (including the one used in our implementation) contain types which are indexed by values, such as Eq $x y$, the type proofs that $x$ and $y$ are equal.

The Helium heuristics for permuting function arguments and tuple elements can be adapted to permute type indices: if permuting the indices of an indexed type removes the error paths from the replay graph, we suggest a fix rearranging them to the user.

\subsection{Error-tolerant typing}

We now describe modifications we made in order to make higher-order unification error-tolerant: that is, able to con- 


\section{$\Delta \widehat{\mapsto} \Delta^{\prime}$ Error-tolerant unification}

These replace the corresponding rules from $\mapsto$. All other rules from $\mapsto$ are present in unaltered form.

$$
\begin{array}{rr}
\Delta, \alpha: T, \Delta^{\prime}, ? \forall \Gamma \cdot \alpha{\overline{x_{i}}}^{i} \equiv t \widehat{\mapsto} \Delta, \Delta^{\prime}, \alpha:=t^{\prime}: T & \text { (inversion-errtol) } \\
& \text { if }{\overline{x_{i}}}^{i} \text { is linear on } \mathrm{FV}(t), \alpha \notin \mathrm{FMV}\left(\Delta^{\prime}, t\right) \text { and } \cdot \mid \Delta, \Delta^{\prime} \vdash \lambda{\overline{x_{i}}}^{i} \cdot t \rightsquigarrow t^{\prime}: T \\
\Delta, \forall \Gamma .(s: T) \equiv(t: T) \hat{\mapsto} \Delta \text { if } s \text { and } t \text { have different rigid head symbols } & \text { (rigid-fail-errtol) } \\
\Delta, \alpha: T, \Delta^{\prime}, ? \forall \Gamma \cdot \alpha{\overline{x_{i}}}^{i} \equiv t \widehat{\mapsto} \Delta, \Delta^{\prime}, \alpha:=\perp: \perp \text { if } \alpha \text { occurs rigid-strong in } t & \text { (fr-occurs-errtol) } \\
\Delta, ? \forall \Gamma . \alpha{\overline{d_{i}}}^{i} \equiv t \widehat{\mapsto} \Delta, \alpha:=\perp: \perp \text { if } \mathrm{FV}^{\text {rigid }}(t) \not \subset \mathrm{FV}\left({\overline{d_{i}}}^{i}\right) & \text { (prune-fail-errtol) }
\end{array}
$$

\section{New and replacement typing rules}

We add a synthesis rule for $\perp$, and an extra side-condition avoiding $\perp$ in with well-typed terms and definitional equality.

$$
\begin{aligned}
& \Gamma \vdash t \rightsquigarrow u \Leftarrow T \\
& \Gamma \vdash s \rightsquigarrow u \Leftarrow T \\
& \Gamma \vdash t \rightsquigarrow u \Leftarrow T \\
& \text { (bot-synth) } \frac{}{\Gamma \mid \Delta \vdash t \rightsquigarrow \perp \Rightarrow \perp} \quad \text { (hastype-errtol) } \frac{u \text { does not contain } \perp}{\Gamma \vdash t: T} \\
& \text { (defeq-errtol) } \frac{u \text { does not contain } \perp}{\Gamma \vdash s={ }_{\beta \delta \eta} t: T}
\end{aligned}
$$

Figure 10: Modified error-tolerant unification, typing and normal forms.

tinue with unification even after an error has been encountered and it is known there is no solution for a certain metavariable. Sometimes, the repair to a type error may modify or delete code causing other errors, so we do not want the programmer to only see the first error encountered, as it may be a symptom of a larger problem.

The core idea is to introduce an explicit error value into our language. Thus, when a metavariable has no solution, we simply assign it an error value and proceed normally.

The modifications to our unification algorithm are described below, with the full details in Figure 10. The errortolerant $\widehat{\mapsto}$ rewrite relation is assumed to be identical to $\mapsto$ except for the changes we highlight. Notably, we never have $\Delta \widehat{\mapsto} \perp$, allowing us to proceed even after errors are discovered.

\subsubsection{Rigid-rigid mismatches}

Any time a rigid-rigid conflict is encountered (i.e. mismatched constructors), unmodified unification throws an error. Instead, we simply take no action, generating no further subproblems from the given equation.

Because rigid-rigid expansion is defined in the exact same way as derived edges in our replay graph, unmodified unification encounters a rigid-rigid error if and only if two disjoint constructors are connected in our final replay graph. We can continue as if the constraint were solved, knowing that the error is reflected in the graph and will be detected.

\subsubsection{An explicit error value}

To model failure in other cases, we adopt the concept of the explicit error, as presented by in counter-factual typing [5, 24].

We introduce the syntactic form $\perp$, which can be used in place of values, heads, etc. As an implementation detail, we annotate $\perp$ with a string describing the context of failure, allowing us to recover useful information during error message generation.

When we encounter a flex-rigid or flex-flex equation with no solutions, such as in an occurs-check, we can assign $\perp$ to the variable whose value we are trying to find, and proceed with unification as if a value had been found.

Moreover, when a value fails to type-check, when generating its normal form, we replace the ill-typed portion with $\perp$. Replacing only the ill-typed portion of a term is made easy through our unified typing and normalization relation. By doing this, we prevent our algorithm from getting stuck where the unmodified algorithm would.

The modified rules for this can be seen in Figure 10. As presented, the rules are not syntax-directed, but in an implementation, we avoid the (bot-synth) rule unless no other rules apply. Since any term can be typed with normal form $\perp$, we add an extra condition to our definitional equality, so that different ill-typed terms are not treated as equal. 


\subsection{Correctness of replay graphs and error-tolerance}

Here, we sketch the proof that the success or failure of unification is not changed by our replay graph and errortolerant modifications.

We take as an assumption the correctness and uniqueness of solutions from the unmodified unification algorithm. That is, if $\Delta_{1} \mapsto^{*} \Delta_{n}$ and $\Delta_{1} \mapsto^{*} \Delta_{k}$, and $\Delta_{n}$ and $\Delta_{k}$ are both final, then either $\Delta_{n}$ and $\Delta_{k}$ are both solved containing equal solutions, or both represent failure (stuck or $\perp$ ). Correctness for similar algorithms is sketched by Abel and Pientka, Gundry and McBride [15, 22].

First, we state our main result:

Theorem 1. Suppose $\Delta_{n}$ is a solved metacontext. Then $\Delta_{1} \mapsto^{*} \Delta_{n}$ if and only if $\Delta_{1} \widehat{\mapsto}^{\star} \Delta_{n}$ with replay graph $G_{n}$ containing no error paths.

To prove the "only if" direction, we first establish that our algorithm does not fail on satisfiable problems. The can be proved using straightforward induction. Intuitively, the result holds because our algorithm's modifications only arise when unification fails.

Lemma 1. If $\Delta_{1} \mapsto^{\star} \Delta_{n}$, where $\Delta_{n}$ is not $\perp$, then $\Delta_{1} \widehat{\mapsto}^{\star} \Delta_{n}$.

The final step in the "only if" direction is to prove that the resulting graph never contains an error when unmodified unification succeeds.

Lemma 2. Suppose $\Delta_{1} \mapsto^{*} \Delta_{n}$ and $\Delta_{1} \widehat{\mapsto}^{*} \Delta_{n}$ with replay graph $\mathcal{G}_{n}$, where $\Delta_{n}$ is a successful solution to $\Delta_{1}$. Let $\sigma$ be the solution substitution implied by $\Delta_{n}$. Then, if $\mathcal{V}(s)$ has a path to $\mathcal{V}(t)$, then $\sigma(s){ }_{\beta \delta \eta} \sigma(t): T$ for some type $T$.

Proof. By strong induction on the length of the path.

If the path has length one, then it is a single edge $E$. If the edge was added from a problem or a solution, then the result follows from the correctness of unmodified unification. Otherwise, the edge was added after a substitution in a raw term, meaning the equality holds by definition.

The inductive step is easily achieved: we divide any path of length $n>1$ into a paths of length $n-1$ and 1 , apply our hypothesis to each sub-path, along with the transitivity of $=_{\beta \delta \eta}$.

Proving the "if" direction is slightly more involved, since our algorithm does behave differently when errors are encountered. We instead prove the contrapositive: any time the unmodified unification fails, the error-tolerant algo- rithm also fails. We prove separate lemmas for the cases when unmodified produces $\perp$ or gets stuck.

The unmodified algorithm produces $\perp$ either by a rigid-rigid mismatch, or by an occurs-check/pruning failure. Our algorithm records these errors in the graph, or by assigning a variable $\perp$, giving us the following lemma.

Lemma 3. Suppose $\Delta_{1} \mapsto^{\star} \Delta_{n}$, where $\Delta_{n}$ is $\perp$. Then $\Delta_{1} \widehat{\mapsto}^{*} \Delta_{m}^{\prime}$ with replay graph $\mathcal{G}_{m}$, where one of the following holds:

- $\mathcal{G}_{m}$ contains an error path.

- A solution in $\Delta_{m}$ contains $\perp$.

Finally, we must deal with the cases where unification fails not by producing $\perp$, but by reaching an unsolved state where no other rules apply. This happens when a typing or definitional-equality side-condition fails, or when no problems are in the pattern fragment. Since we produce a term containing $\perp$ when type-checking fails, we obtain the following lemma, which is the final piece needed to prove our main result.

Lemma 4. Suppose $\Delta_{1} \mapsto^{\star} \Delta_{n}$, where $\Delta_{n}$ is stuck. Then $\Delta_{1} \widehat{\mapsto}^{*} \Delta_{n}$ with replay graph $\mathcal{G}_{n}$, where one of the following holds:

- $\mathcal{G}_{n}$ contains an error path

- A solution in $\Delta_{n}$ contains $\perp$

- $\Delta_{m}$ is stuck

Additionally, these lemmas show that each specific error found using $\mapsto$ is also found using $\widehat{\mapsto}$, since they behave identically until immediately before $\mapsto$ reaches $\perp$.

\section{Counter-factual unification}

In the above sections, type graphs are used only to provide replays of unification, and do not direct the unification process. Thus, they still contain some bias: the order in which we solve constraints can affect the results.

As a solution, we present counter-factual unification: a modification to unification which seeks to reduce bias by exploring solutions arising from subsets of the initial constraint-set. The main idea is that, when we find a solution for a variable, we proceed with unification both with the solution, and as if the solution had never been found. This allows us to see conflicting potential solutions, without being biased towards which one was generated first.

To accommodate this process with relative efficiency, we utilize the choice calculus [25] for compact representation of multiple constraint sets, as well as the modifi- 


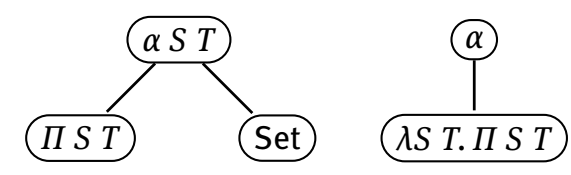

(a) Possible (partial) replay graph for $\{\forall S, T . \alpha S T \equiv \Pi S T, \forall S, T . \alpha S T \equiv$ Set $\}$. The solution $\alpha:=\lambda S T$. $\Pi S T$ is only generated when we process the edge from $\alpha$ to $\Pi S T$ first, showing bias. $\alpha S T$ is on an error path, but $\alpha$ is not, giving us little information for our heuristics to guess its correct value.

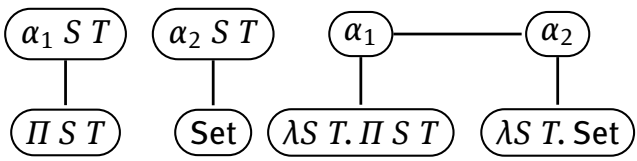

(b) If instead, we use different metavariables for our two constraints, with a separate constraint unifying them, we have the two conflicting solutions on one error path.

Figure 11: Bias and a counter-factual solution.

cations to unification needed for solving these. We then prove that our modified algorithm halts, and produces equivalent results to unmodified unification.

Here, we provide the motivation and theory for counter-factual dependently-typed unification. Our implementation does not yet utilize these techniques, but we anticipate that future work will integrate them into the implementation.

\subsection{Motivation}

While there are several potential sources of bias, a particularly problematic one is in substitutions. The order in which constraints are solved in dynamic higher-order unification matters, and when a potential value is found, it is substituted into all other problems as the "actual" value, and future conflicting values are seen as rigid errors.

The inability of type graphs to handle this case arises from the need to unify arbitrary terms containing metavariables, particularly those containing function applications.

Consider the following unsatisfiable constraint set, whose replay graph is given in Figure 11:

$$
\left\{P_{1}=\forall S, T . \alpha S T \equiv \Pi S T, \quad P_{2}=\forall S, T . \alpha S T \equiv \text { Set }\right\}
$$

In normal unification, if we solve $P_{1}$ first, we define $\alpha$ := $\lambda S T$. $\Pi S T$. We substitute the new value into $P_{2}$, and after evaluation, get $\Pi S T \equiv$ Set. This update to the value of $\alpha S T$ is recorded. However, in our type graph, our node for $\alpha$ will only have an edge to $\lambda S T$. $\Pi S T$. We have a path from $\Pi S T$ via $\alpha S T$ to Set, but the node for $\alpha$ itself occurs on no error paths. We are never able to see that $\lambda S T$. Set is a potential solution for $\alpha$.

The opposite problem happens if we begin with the second constraint, exposing bias in our solving procedure. Even with type graphs, the order in which we solve constraints affects the error messages. Ideally, we want all conflicting values for $\alpha$ to be connected to it, so that we can present them as possible solutions, or use heuristics to determine which one is likely to be correct.

As a potential solution, suppose that we decomposed the above constraint set into the following:

$$
\begin{gathered}
\left\{P_{1}=\forall S, T . \alpha_{1} S T \equiv \Pi S T,\right. \\
\left.P_{2}=\forall S, T . \alpha_{2} S T \equiv \text { Set, } \quad P_{3}=\alpha_{1} \equiv \alpha_{2}\right\}
\end{gathered}
$$

As we can see in Figure 11, we now obtain an error path containing both $\alpha_{1}$ and $\alpha_{2}$.

We formalize the intuition behind this procedure, showing how to solve such problems. Specifically, we adapt the ideas from counter-factual typing [5], which in turn is based on the Choice Calculus [25]. These provide a method to counteract bias by examining solutions that arise if we had never given a variable its definition.

\subsection{A choice calculus}

\subsubsection{Choice operations and terminology}

In order to facilitate counter-factual solving, we need a way to compactly represent multiple possible solutions to a unification problem. To allow this, we augment our language with a form for named choice expressions: $t::=\ldots \mid C\left\langle t_{1}, t_{2}\right\rangle$, where $C$ is an identifier. If a term $t$ contains choices $C_{1}, \ldots, C_{n}$, we say that these are the dimensions of the term, denoted by $\operatorname{dim}(t)$.

A choice is effectively a pair combined with a label. However, the choice calculus is equipped with an equational theory that gives it meaning beyond that of simple tuples. For example, $x$ and $C\langle x, x\rangle$ are equivalent expressions. The named choices allow us to represent variations efficiently within large expressions. For example, the application $C\left\langle f 0 x_{1} \ldots x_{n} 2, f 1 x_{1} \ldots x_{n} 3\right\rangle$ can be more compactly represented as $f C\langle 0,1\rangle x_{1} \ldots x_{n} C\langle 2,3\rangle$.

In order to avoid conflicts between solutions in different choice dimensions, we qualify metavariables by the choices inside which they occur [24]. A qualifier string $q$ is some (possibly empty) sequence of qualifiers, each of which is either $C^{L}$ (left) or $C^{R}$ (right), where $C$ is the name of a choice. We use $t_{q}$ as shorthand for adding the qualifier $q$ to all metavariables in $t$. 


$$
\begin{aligned}
& s, t, S, T::=\ldots|C\langle s, t\rangle| \alpha_{q} \\
& d, e::=\ldots \mid C\langle d, e\rangle \\
& \mathrm{E}::=\ldots|C\langle\mathrm{E}, t\rangle| C\langle v, \mathrm{E}\rangle \\
& q::=\cdot\left|q C^{L}\right| q C^{R} \\
& C\langle s, t\rangle \cdot C\left\langle e_{1}, e_{2}\right\rangle{\overline{e_{i}}}^{i} \rightarrow C\left\langle s \cdot e_{1}, t \cdot e_{2}\right\rangle \cdot{\overline{e_{i}}}^{i} \\
& t \cdot C\left\langle e_{1}, e_{2}\right\rangle{\overline{e_{i}}}^{i} \rightarrow \operatorname{hoist}(C, t) \cdot C\left\langle e_{1}, e_{2}\right\rangle{\overline{e_{i}}}^{i} \\
& C\langle s, t\rangle \cdot e{\overline{e_{i}}}^{i} \rightarrow C\langle s, t\rangle \cdot \operatorname{hoist}(C, e){\overline{e_{i}}}^{i} \\
& \text { (meta-qualL) } \frac{\Gamma \mid \Delta \vdash \alpha_{q} \rightsquigarrow \alpha_{q} \Rightarrow T}{\Gamma \mid \Delta \vdash \alpha_{q C} \rightsquigarrow \alpha_{q C^{L}} \Rightarrow C_{L} T} \\
& \text { (meta-qualR) } \frac{\Gamma \mid \Delta \vdash \alpha_{q} \rightsquigarrow \alpha_{q} \Rightarrow T}{\Gamma \mid \Delta \vdash \alpha_{q C^{R}} \rightsquigarrow \alpha_{q C^{R}} \Rightarrow C_{R} T} \\
& \text { (ch-hoist1) } \frac{\Gamma \mid \Delta \vdash C\langle s, t\rangle \rightsquigarrow u \Leftarrow \operatorname{hoist}(C, T)}{\Gamma \mid \Delta \vdash C\langle s, t\rangle \rightsquigarrow u \Leftarrow T} \\
& \text { (ch-hoist2) } \frac{\Gamma \mid \Delta \vdash \operatorname{hoist}(C, t) \rightsquigarrow u \Leftarrow C\langle S, T\rangle}{\Gamma \mid \Delta \vdash t \rightsquigarrow u \Leftarrow C\langle S, T\rangle} \\
& \Gamma \mid \Delta \vdash s \rightsquigarrow u \Rightarrow S \\
& \text { (ch-synth) } \frac{\Gamma \mid \Delta \vdash t \rightsquigarrow v \Rightarrow T}{\Gamma \mid \Delta \vdash C\langle s, t\rangle \rightsquigarrow C\langle u, v\rangle \Rightarrow C\langle S, T\rangle} \\
& \Gamma \mid \Delta \vdash s \rightsquigarrow u \Leftarrow S \\
& \text { (ch-check) } \frac{\Gamma \mid \Delta \vdash t \rightsquigarrow v \Leftarrow T}{\Gamma \mid \Delta \vdash C\langle s, t\rangle \rightsquigarrow C\langle u, v\rangle \Leftarrow C\langle S, T\rangle}
\end{aligned}
$$

Figure 12: Choice syntax, semantics and typing.

For each choice identifier $C$, we define left and right projections, $C_{L}$ and $C_{R}$. These map every occurrence of choice $C$ to their left or right sides, respectively, while stripping metavariables of any $C$ qualifiers. We allow these projections to be applied to arbitrary terms, constraints, and metacontexts, where all occurrences of a choice are replaced with the left or right variant respectively. Similarly, we define a projection Factual which maps all choices to their left-hand side.

One final operation that we will make use of is hoisting. This allows us to take a term containing multiple choices in the same dimension, and lift them to the top level. When we hoist a term $t$ with respect to choice $C$, denoted hoist $(C, t)$ we produce the term $C\left\langle C_{L} t, C_{R} t\right\rangle$. Note that hoist, $C_{L}, C_{R}$ and Factual are operations in the metalanguage, rather than constructs in the language itself.

For example, consider the following term:

$$
t=C_{1}\left\langle C_{2}\langle x, y\rangle, z\right\rangle
$$

The projection $C_{1 L} t$ gives us $C_{2}\langle x, y\rangle$, and $C_{1 R} t$ gives $z$. Similarly, $C_{2 L} t$ gives $C_{1}\langle x, z\rangle$, and $C_{2 R} t$ gives $C_{1}\langle y, z\rangle$. To get the left side of all choices, we can write Factual $t$ to obtain $x$. To bring $C_{2}$ as the top level choice, we use hoist $\left(C_{2}, t\right)$ to obtain the equivalent expression $C_{2}\left\langle C_{1}\langle x, z\rangle, C_{1}\langle y, z\rangle\right\rangle$. Hoisting $C_{1}$ is a valid operation, but returns $t$ unchanged since $C_{1}$ is already the top-level choice.

\subsubsection{Semantics and typing}

The semantics and typing rules for choice are in Figure 12. To apply an eliminator to a head, where both have a top level choice in dimension $C$, we simply return the choice between applying the left eliminator to the left head and the right eliminator to the right head. If only one of the head and eliminator is a choice, or they are choices in different dimensions, we can hoist to move the same choice to the top level.

The type rules work in much the same way. In (metaqualL) and (meta-qualR), we synthesize the type for a qualified type variable by finding the type for the unqualified variable, and projecting left or right according to the qualifier tag. The remaining rules account for choices in types and terms. There are two steps to checking. First, we must ensure the term and the type we are checking it against have the same top-level choice. We do this with (ch-hoist1) and (ch-hoist2), using hoist to bring any nested choices to the top-level. Then, to check a choice expression against a choice type in (ch-check), we simply check each dimension of the choice separately. To synthesize the type for a choice expression, we synthesize a type for each dimension, then pair these with the expression's choice label.

Intuitively, choices represent the set of terms obtained from all possible combinations of projections on each dimension. There are $2^{n}$ constraints represented by a term containing $n$ distinct choice labels, assuming each choice has two distinct variants. The counting is more complicated when choice labels are nested, but a potentially exponential set of values is in this way compactly represented. 


\subsection{Counter-factual solving}

In Figure 13 we provide the definition for our counterfactual unification rewrite relation, denoted by $\widetilde{\mapsto}$. We assume that all rules not explicitly listed are identical to $\widehat{\mapsto}$.

The main idea of counter-factual solving is that whenever unmodified unification generates a substitution $\alpha:=$ $t$, we instead generate $\alpha:=C\left\langle t, \alpha^{\prime}\right\rangle$, where $C$ and $\alpha^{\prime}$ are fresh, so that the solver can also proceed as if no value were ever given to $\alpha$. We refer to $t$ as the factual case, and to $\alpha^{\prime}$ as the counter-factual case, since they present the solutions for whether the constraint was or was not present.

Traditional counter-factual typing is defined as part of the typing judgement. However, due to the complexities introduced by dependent-types, we instead use choice only during the unification process.

\section{Defining metavariables}

When we find a solution $\alpha:=t$ in a problem $P$, we instead generate $C\left\langle t_{C^{L}}, \alpha^{\prime}\right\rangle$ as a solution, where $\alpha^{\prime}$ is a fresh metavariable. Here, $t_{C^{L}}$ is $t$ with the qualifier $C^{L}$ added to all metavariables occurring within $t$. This ensures that, if solutions to $\alpha^{\prime}$ contain metavariables in $t$, their solutions will not conflict.

\section{Solving equations with choice}

An equation with a choice point represents a set of separate equations, which we would like to solve separately, without solutions to one equation affecting the others. This problem is solved by considering qualified type variables as distinct unless they are identical variables with identical qualifiers. Solutions for qualified variables are generated, and the full solution for variable $\alpha$ can be reconstructed from the qualified solutions through completion [26], where it is assigned a value whose choices correspond to the choices of each qualified solution. However, our treatment of choices within replay graphs means that we do not need to perform completion to generate error messages. We elaborate on this in Section 7.4.

Solving equations involving choice can be achieved through manipulating those choices. The equation $C\langle s, t\rangle \equiv C\left\langle s^{\prime}, t^{\prime}\right\rangle$ is easily solved by decomposition into $s \equiv s^{\prime}, t \equiv t^{\prime}$. When only one side is a choice in dimension $C$ at the top level, we can apply hoist, which will move any occurrences of $C$ to the top level. If one side is a term $t$ containing no choices, hoist $(C, t)$ produces $C\langle t, t\rangle$. Note that we must hoist here, but we wish to hoist only when necessary, since it can duplicate large parts of the term being hoisted.

\subsubsection{Performance}

When we apply counter-factual solving, we see an exponential explosion in the number of problems and the size of the context, in what is already a potentially slow algorithm. While the choice calculus helps keep this number smaller, most choices will ultimately be pointless, as we will find equal values for both sides of choice expressions.

A possible solution is as follows. When we split a constraint involving a choice, as described above, we mark newly generated equations for the right-side of the choice as pending on the failure of the left-hand side. Thus, if the unification problems involving the choice all succeed, we never explore the counter-factual cases.

\subsection{Counter-factual replay graphs}

The use of counter-factual typing helps generate potential solutions for ill-typed metavariables. However, we still wish to take advantage of the information available to us from type graphs, and the heuristics for diagnosing error locations.

Our solution is simple: when we generate a definition $\alpha:=C\left\langle t, \alpha^{\prime}\right\rangle$, we add an equality edge between $\alpha$ and $\alpha^{\prime}$. For other occurrences of choice, we simply add an undirected edge between the vertices for the two sides of the choice. The intuition behind this is that, when a program is well typed, both dimensions of a choice for a metavariable's solution should agree. Disagreements between the factual and counter-factual cases become error paths in our program.

Additionally, counter-factual solving can be used in a repair heuristic. Whenever we define $\alpha:=C\left\langle t, \alpha^{\prime}\right\rangle$, we have an edge from $\alpha$ to $t$, and one from $\alpha$ to $\alpha^{\prime}$. If we remove the edge from $\alpha$ to $t$ (as well as its dependent edges), and the new graph has fewer inconsistencies, then we can suggest the value of $\alpha^{\prime}$ as a likely fix for the value of $\alpha$.

\subsubsection{Redundancy}

Another practical issue with counter-factual unification is that our type graphs are now redundant. Since the factual case behaves as unmodified unification does, each error is reflected twice in our type graph: once for the error in the factual case, and once in the disagreement between the factual and counter-factual cases.

To avoid this redundancy, an implementation can keep in its context the string of choice dimensions that have been currently assumed. For example, when solving 


\section{$\Delta \widetilde{\mapsto} \Delta^{\prime} \quad$ Counter-factual unification}

We assume that all rules from $\hat{\mapsto}$ are present, except for those that are replaced below.

\section{New rules}

These rules work in addition to those for $\hat{\mapsto}$.

$\Delta, \forall \Gamma . C\langle s, t\rangle: T \equiv C\left\langle s^{\prime}, t^{\prime}\right\rangle: T^{\prime} \sim \widetilde{\mapsto} \Delta, \forall \Gamma . s: T \equiv s^{\prime}: T^{\prime}, \forall \Gamma . t: T \equiv t^{\prime}: T^{\prime}$

(cf-decomp)

$\Delta, \forall \Gamma . C\langle s, t\rangle: T \equiv t^{\prime}: T^{\prime} \sim \widetilde{\mapsto} \Delta, \forall \Gamma . C\langle s, t\rangle: T \equiv \operatorname{hoist}\left(C, t^{\prime}\right): T^{\prime}$

(cf-hoist)

\section{Modified rules}

These rules replace their corresponding versions from $\hat{\mapsto}$

$$
\Delta, \alpha: T, \Delta^{\prime}, ? \forall \Gamma . \alpha{\overline{x_{i}}}^{i} \equiv t \widetilde{\mapsto} \Delta, \Delta^{\prime}, \alpha:=C\left\langle t_{C^{L}}^{\prime}, \alpha^{\prime}\right\rangle: T
$$

if ${\overline{x_{i}}}^{i}$ linear on $\mathrm{FV}(t), \quad \alpha \notin \mathrm{FMV}\left(\Delta^{\prime}, t\right), \quad \cdot \mid \Delta, \Delta^{\prime} \vdash \lambda{\overline{x_{i}}}^{i} . t \rightsquigarrow t^{\prime}: T, \quad \alpha^{\prime} \notin \mathrm{FMV}\left(\Delta, \Delta^{\prime}\right), \quad C \notin \operatorname{dim}\left(\Delta, \Delta^{\prime}\right)$

$\Delta, \alpha: \Pi \Phi . T, \Delta^{\prime}, ? \forall \Gamma . \alpha{\overline{x_{i}}}^{i} \equiv \alpha{\overline{y_{i}}}_{i}^{i} \sim \Delta, \Delta^{\prime}, \beta: \Pi \Phi . T$ if ${\overline{x_{i}}}^{i}$ matches ${\overline{y_{i}}}^{i}$ on $\Psi \subset \Phi$,

(intersection-cf)

$$
\alpha:=C\left\langle(\lambda \Phi . \beta \Psi)_{C^{L}}: \Pi \Phi . T, \alpha^{\prime}\right\rangle \operatorname{FV}(T) \subset \operatorname{vars}(\Psi), \alpha^{\prime} \notin \operatorname{FMV}(\Delta, \beta: \Pi \Phi . T), C \notin \operatorname{dim}\left(\Delta, \Delta^{\prime}\right)
$$

Figure 13: Counter-factual unification rules.

$C_{1}\left\langle C_{2}\left\langle T_{1}, T_{2}\right\rangle, T_{3}\right\rangle \equiv S$, we would store the string $C_{1} \overline{C_{2}}$ when solving the equation $T_{2} \equiv S$. Then, in our graph, we make each generated edge dependent on the solution that first introduced the choices stored in the context. With our example above, if $C_{1}$ was a fresh choice generated by the solution $\alpha:=C_{1}\left\langle S^{\prime}, \alpha^{\prime}\right\rangle$, and $C_{2}$ from $\beta:=C_{2}\left\langle S^{\prime \prime}, \beta^{\prime}\right\rangle$, then the edge $\left\{T_{2}, S\right\}$ would be dependent on the edges $\left\{\alpha, S^{\prime}\right\}$ and $\left\{\beta, S^{\prime \prime}\right\}$.

The intuition behind this is that, when diagnosing an error, if we delete an edge from the factual case of a solution, we should also delete the edges that were made under the assumption that the factual case held. Each intermediate constraint will still be represented in the graph, but only in the counter-factual case for the deleted edge.

\subsection{Correctness}

Here we establish the relationship between counterfactual unification and our error-tolerant algorithm. Our main result is as follows:

Theorem 2. If $\Delta_{1} \widehat{\mapsto}^{\star} \Delta_{m}$ and $\Delta_{1} \widetilde{\mapsto}^{\star} \Delta_{n}^{\prime}$, where $\Delta_{m}$ and $\Delta_{n}^{\prime}$ are both final, then $\Delta_{m}$ is solved if and only if $\Delta_{n}^{\prime}$ is.

We first note that we can simulate error-tolerant unification in our counter-factual system. This can be proved by induction, utilizing the fact that the only modified rules keep the original result in the left-hand side of the choice. The counter-factual run may take more steps, since some steps are required to decompose choices.
Lemma 5. If $\Delta_{1} \widehat{\mapsto}^{\star} \Delta_{m}$, then $\Delta_{1} \widetilde{\mapsto}^{\star} \Delta_{n}^{\prime}$, where $m \leq n$ and Factual $\left(\Delta_{n}^{\prime}\right)=\Delta_{m}$

Conversely, we can recover an error-tolerant run from any counter-factual run. The proof is similar to Lemma 5, with the added detail that the (unif-choice) rule produces changes that are completely erased by Factual.

Lemma 6. If $\Delta_{1} \widetilde{\mapsto}^{\star} \Delta_{n}^{\prime}$, then $\Delta_{1} \widehat{\mapsto}^{\star} \Delta_{m}$, where $m \leq n$ and Factual $\left(\Delta_{n}^{\prime}\right)=\Delta_{m}$.

Finally, we strengthen Theorem 2 to show that counterfactual solving terminates whenever normal unification does.

Theorem 3. If $\Delta_{1} \widehat{\mapsto}^{\star} \Delta_{n}$, where $\Delta_{n}$ is solved or stuck, then there exists some $m$ such that $\Delta_{1} \mapsto^{\star} \Delta_{m}^{\prime}$, where $\Delta_{m}^{\prime}$ is either solved or stuck.

Proof. Gundry and McBride [22] give an intuition that $\mapsto$ always progresses towards a solution, and we have shown that $\widehat{\mapsto}$ will reach a solution or failure in finitely many steps whenever $\mapsto$ does. Thus, we assume a well-founded ordering $\prec$ such that if $\Delta \widehat{\mapsto} \Delta^{\prime}$, then either $\Delta$ and $\Delta^{\prime}$ differ only in permutations and symmetry, or $\Delta^{\prime} \prec \Delta$.

We define a new partial order $\sqsubset$ as the smallest partial order where $\Delta^{\prime} \sqsubset \Delta$ when any of the following hold:

$$
\Delta^{\prime} \prec \Delta
$$

$C_{L} \Delta^{\prime} \prec \Delta$ and $C_{R} \Delta^{\prime} \prec \Delta$ for some $C$

$\Delta^{\prime}$ is $\Delta$ with a choice hoisted outwards 
The partial order $\sqsubset$ is well founded. In any descending chain, the number of choice dimensions may increase only if each underlying dimension is strictly descending with respect to $\prec$. So the number of dimensions we can add in a descending $\sqsubset$ chain is limited by the length of descending $\prec$ chains, which are always finite. Likewise, choices can only be hoisted outwards finitely many times.

Finally, we show that if $\Delta \widetilde{\mapsto} \Delta^{\prime}$, then $\Delta^{\prime} \sqsubset \Delta$, or they are equal up to permutation and symmetry. This is immediate for unmodified rules by (1). For (inversion-cf) and (intersection-cf), we introduce a dimension $C$ for our solution, but replace a problem with its solution in each dimension, so our order is respected by (2). For (ch-decomp) we are smaller by (1) because we decompose one problem into two structurally smaller ones $(C\langle s, t\rangle$ is structurally larger than both $s$ and $t$ ). For (ch-hoist) we are smaller by (3) because a choice is hoisted outwards.

\section{Results and discussion}

In order to evaluate our techniques, we combined the implementations of Helium, LambdaPi, and Gundry-McBride Unification. A few Helium heuristics, such as the application and permutation heuristics, were transferred to our system, along with our index-permutation heuristic specific to dependent-types. The source code of the implementation is publicly available [27].

We present some simple programs containing type errors and compare our generated error messages with those from roughly equivalent programs in Agda and Idris. For brevity, we omit the code for the Agda and Idris versions.

An issue with solving is that several errors are often identified, where only one should be. For the sake of these examples, we simplify the messages presented to a single error, in order to highlight the repair heuristics of our system. We discuss the system's limitations in Section 8.2.

\subsection{Results}

\subsubsection{Too many arguments}

If we give a function too many arguments, we can give a helpful hint informing the user of the expected number. From the replay graph, we can look at the context of the error and find the entire function type, seeing that it takes three arguments. Both Agda and Idris only report the error for the partially-applied function, and Agda does not even warn the user that Nat is not a function type.
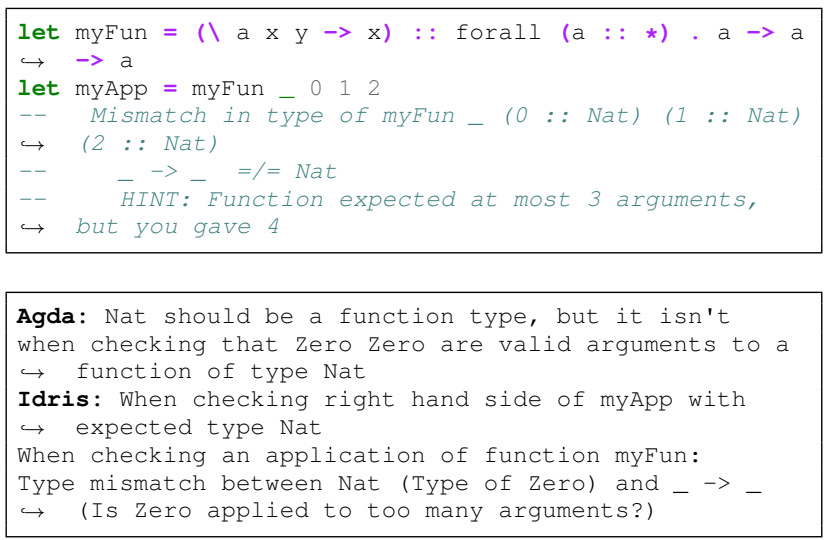

\subsubsection{Too few arguments}

When too few arguments are given, our repair heuristics are able to suggest both the types and positions of arguments which must be added in order to create a well-typed function call. This is done by repeatedly adding arguments with metavariable types to the application in the replay graph, stopping either when the type graph becomes consistent, or when the return type is no longer an arrow type. Agda and Idris only inform the user that the actual type of the expression is a function type, describing its mismatch with the result type.

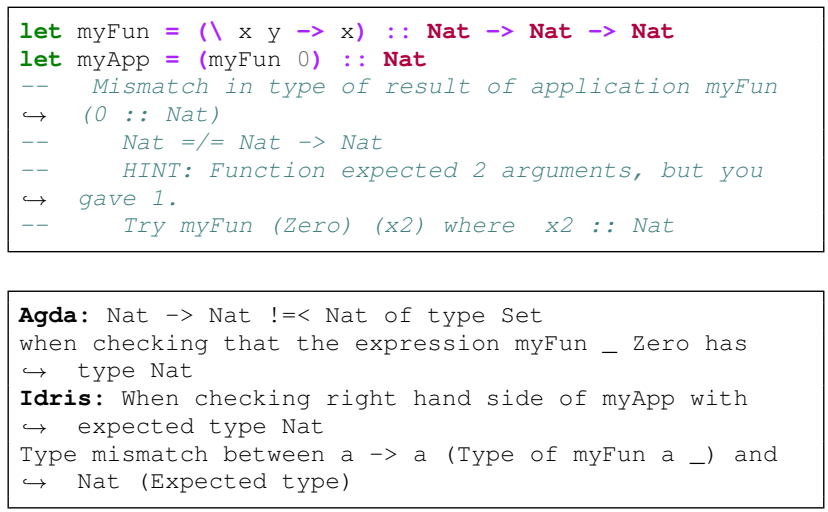

\subsubsection{Arguments in the wrong order}

As with too few arguments, our repair heuristics can use a similar procedure to try different permutations of arguments, suggesting the one that is likely to resolve the error. 


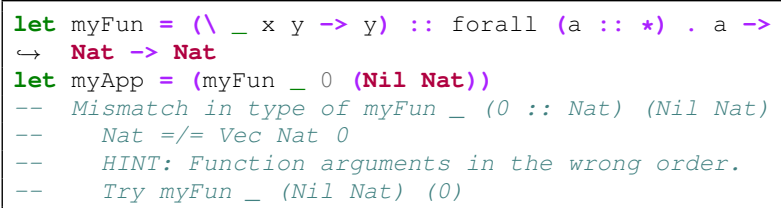

Agda and Idris report a mismatch, but provide no hint that the arguments should be swapped.

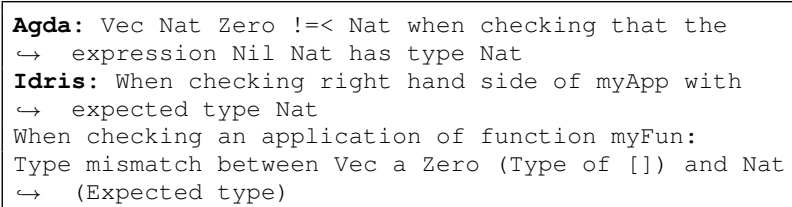

\subsubsection{Dependent-type error}

Here we show the error message arising from a classic, dependent-type specific mistake: reversing the indices in the type of an equality proof. This example can be fixed by reversing the type signature or applying a proof of equality's symmetry.

We are able to apply an isomorphism heuristic, a variant of the permutation heuristic that permutes dependenttype indices. This allows us to see that the two conflicting types are isomorphic to one another, prompting the user to rearrange them.

For brevity, we use plus in place of its body in our error messages.

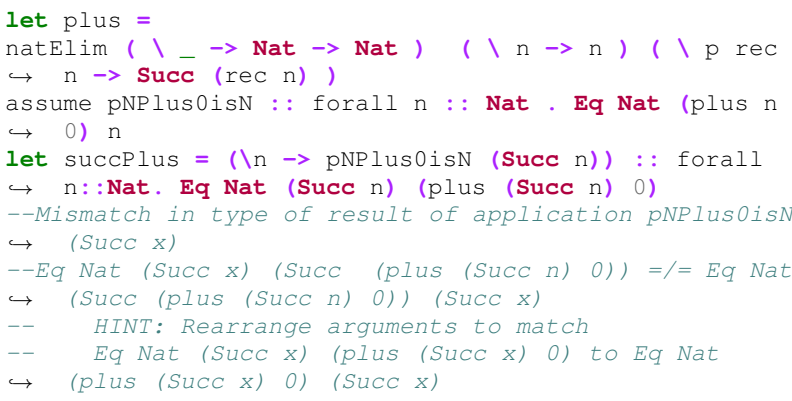

Agda and Idris report the mismatch but are unaware that a swap would fix it.

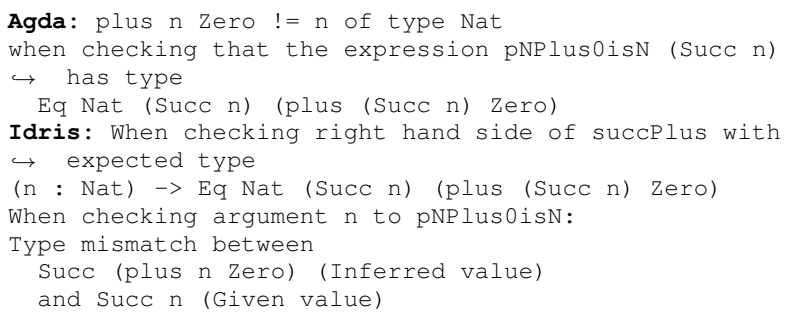

\subsection{Limitations}

While we are able to provide helpful hints in many cases, the system is not without its flaws. Our results are promising, but the issues below will require solutions before our techniques are ready for integration into a mainstream language.

\section{Error redundancies}

Our compiler sometimes reports the same message multiple times. In particular, when reporting possible error locations, several constraints contributing the same conflicting value are reported.

\section{Dependent edges in repair heuristics}

Our compiler uses replay graphs to generate derived edges from initial constraints. However, many heuristics involve removing and adding edges in the graph. While it is easy to add implicit edges from constructor applications, the situation is complicated for dependent edges generated during unification. When modifying the graph in a heuristic, removing the edges for an initial edge deletion is simple, but generating dependent edges when new edges are added is not. Re-running unification may be too costly for practical purposes. Because of this, some heuristics generate "false positives," where a suggestion is given that will not actually resolve the type errors, because dependent edges cause inconsistencies that are not immediately apparent in the graph. Some simple heuristics, such as disallowing identity permutations as hint suggestions, help with these difficulties.

\section{Controlling evaluation}

The error messages our compiler generates are often long and unwieldy. This is because Gundry and McBride's implementation, and hence ours, is aggressively strict in its name lookup strategy. As a result, names are prematurely replaced by their values. The examples above involving plus would be much more readable if the actual printed used plus in place of its body, as we present it.

The issue of lazy versus strict evaluation, and of choosing when to replace identifiers by their values, seems critical for the generation of readable error messages, and we suspect addressing this issue will be a project unto itself. 


\subsection{Future work}

In addition to resolving the above limitations, we highlight a few more potential research topics.

\section{Heuristics}

We have presented a framework, which allows for the analysis of type-errors using heuristics on type graphs. While we have adapted a few Helium heuristics to our framework, there is room for heuristics specifically targeted at dependent-types. A Helium-style collection of heuristics could be manually collected, or future work could incorporate the Bayesian heuristics from SHErrLoc [28].

\section{Empirical evaluation}

An important aspect of future work is to empirically evaluate the accuracy of our error messages, and the speed at which we can generate them. Such an evaluation was not feasible for this work: dependently-typed code is rare in the wild, and that which is published generally contains no type errors. On top of this, our implementation is for a small core calculus, missing many features of real code.

One possible method of evaluation would be to instrument Idris or Agda to record error messages, then to have a class of students learning dependent-types use the instrumented compiler. This would provide a reasonably large sample of faulty code, while also providing the potential for students to evaluate the usefulness of messages for beginners.

\section{Interactivity}

Both Agda and Idris feature highly interactive editors, where the programmer leaves holes in their code, and the editor can manipulate code in type directed ways, such as filling in holes or case-splitting variables. When code is type-incorrect, the type graph could be used to suggest hints not only in error messages, but interactively in the editor.

\section{Performance}

Initially, performance is not a major concern: since dependent-types require annotations, each declaration can be type-checked separately, so our techniques could be limited to the specific module, or even specific binding group, that the programmer is currently writing.

That said, the unification algorithm which we adapted was, according to the authors, not tuned for performance, with much needless iteration through the context. A more sophisticated algorithm could yield performance improvements.

Switch combinators were developed for Helium [4] to address the computational costs associated with type graph analysis. Possible future work could examine how these could be adapted to our system.

\section{Alternate constraint solvers}

While our algorithm is based on Gundry and McBride's presentation of unification, there are several different higher-order unification algorithms [15, 19]. For exploratory research, Gundry-McBride unification was ideal. However, other algorithms could be faster, or able to solve a wider set of problems. The concepts we introduce, such as replay graphs and counter-factual solving, can likely be applied to more sophisticated constraint solvers.

\section{Related work}

\subsection{Unification algorithms}

Huet [29] showed that higher-order unification was undecidable in general, but later provided a semi-decision procedure for it [30]. The original pattern unification algorithm was presented by Miller [14]. This was later extended by Abel and Pientka [15] to accommodate dependent pairs and records, as well as to be dynamic, allowing problems outside the pattern fragment so long as they are eventually removed by substitutions from solving variables. Gundry and McBride [22] presented a version of unification tailored towards a practical implementation. The algorithm we present is a direct modification of theirs, along with the version in the thesis of Gundry [21].

A unification algorithm for Coq has been developed to account for universe polymorphism and overloading, as well as to incorporate heuristics to solve some cases outside of the pattern fragment [19]. In Agda, pattern matching was modified to accommodate univalent theories [31], and a new unification algorithm has been added that models unifiers as dependently-typed equivalences [23, 32].

\subsection{Error message improvement}

Our contribution is a refinement of the first author's Master's thesis [33]. To our knowledge, this was the first work to specifically explore error message reporting for 
dependent-types. However, the topic has been studied extensively for Hindley-Milner and Object-Oriented languages.

\section{Reporting strategies}

Haack and Wells [8] introduce the concept of an error slice: a set of program points which contribute to the error. This was one of the first constraint-based methods for error reporting. It is based around the idea that multiple locations should be reported, since the true location of an error can depend on the programmer's desired semantics.

The idea of presenting multiple error locations is extended by Chen and Erwig [5] through a technique known as counter-factual typing. In counter-factual typing, the checker examines atomic expressions and determines what type the expression would need to have in order for the program to type-check. This, in turn, was an extension of the work on variational lambda calculus and error-tolerance [24, 26]. The counter-factual unification and error-tolerant typing we present are an adaptation of this work to dependent-types.

Campora et al. [34] present an alternate method of achieving error-tolerance through pattern-constrained judgments. By introducing explicit typing patterns which specify in which dimensions two types agree, they are able to remove the need for an explicit $\perp$ type in their language.

\section{Helium}

Helium [2-4] is an alternate Haskell compiler, which aimed to improve the quality of error messages, particularly for beginners. At its core was a type-inference algorithm based on the concept of constraint-generation, where constraints are emitted, rather than having a single substitution gradually grow as in Algorithm W [35]. The various strategies for solving these constraint sets can emulate classical inference algorithms [36], as well as allowing for heuristic analysis. A more advanced technique Helium used was the construction of constraint graphs, creating a global representation of the entire type-inference problem to be heuristically analyzed. Our replay graphs are a direct adaptation of Helium's type graphs, and our implementation draws heavily from the public Helium code base [37].

\section{Bias}

Much of the work on error diagnosis seeks to avoid bias. In Helium, type graph help avoid bias within a binding group, but not between binding groups. Counter-factual typing avoids the latter by making it possible to blame an early binding group if a later binding group provides the information to pinpoint the actual mistake, essentially by not making us commit to a particular choice early on in the program. The work by Pavlinovic [38] takes a different approach which seems to scale much better: when a binding group contains a type error, definitions within the same module are inlined until the error location is not simply the location of a particular identifier defined earlier. This may mean that for that binding group the inference process is re-run multiple times, but in practice, a few inlining steps suffice.

\section{SHErrLoc}

An alternate approach to the constraint-based system of Helium is implemented in SHErrLoc [28]. Its constraints are over arbitrary partial orders, and is generic enough to describe Hindley Milner typing, as well as information-flow and data-flow analyses. Constraint solving uses context-free reachability. This approach can typecheck more advanced systems, such as type classes and GADTs [9]. However, it treats the axioms from type-level functions as pre-existing, and it is thus ill-suited to languages with unified types and terms and arbitrary typelevel computations.

\section{Conclusion}

In this work, we have identified the challenges of adapting known functional error-message generation to dependently-typed languages. With replay graphs and counter-factual solving, we have taken the first steps towards improving error messages for dependent-types. Replay graphs were implemented for a small language, and initial evaluation showed that helpful hints could be generated for simple examples.

While our approach has limitations, we have shown that it is possible to use more advanced error generation techniques with dependent-types. By identifying possible approaches, as well as challenges, for dependent-type error generation, we hope to provide a solid foundation for future research on the ease of use of dependently-typed languages.

\section{Acknowledgements}

This work was supported in part by the Natural Sciences and Engineering Research Council of Canada and the Utrecht Excellence Scholarship. 


\section{References}

[1] Barik T., Smith J., Lubick K., Holmes E., Feng J., Murphy-Hill E., Parnin C., Do developers read compiler error messages?, In: Proceedings of the 39th International Conference on Software Engineering (ICSE '17), IEEE Press Piscataway, NJ, USA, 2017, 575-585, DOI: 10.1109/ICSE.2017.59

[2] Heeren B., Hage J., Swierstra S. D., Constraint based type inferencing in Helium, Immediate Applications of Constraint Programming (ACP), 2003, 57, http://www.open.ou.nl/bhr/ ConstraintBasedTI.html

[3] Hage J., Heeren B., Heuristics for type error discovery and recovery, In: Proceedings of the 18th International Conference on Implementation and Application of Functional Languages (IFL'06), Springer-Verlag, Berlin, Heidelberg, 2007, 199-216, DOI:10.1007/978-3-540-74130-5_12

[4] Heeren B. J., Top quality type error messages, Ph.D. thesis, Utrecht University, Utrecht, Netherlands, 2005, IPA Dissertation Series, https://dspace.library.uu.nl/handle/1874/7297

[5] Chen S., Erwig M., Counter-factual typing for debugging type errors, In: Proceedings of the 41st ACM SIGPLANSIGACT Symposium on Principles of Programming Languages (POPL '14), ACM, New York, NY, USA, 2014, 583-594, DOI: $10.1145 / 2535838.2535863$

[6] Norell U., Dependently typed programming in Agda, In: Proceedings of the 4th International Workshop on Types in Language Design and Implementation (TLDI '09), ACM, New York, NY, USA, 2009, 1-2, DOI: 10.1145/1481861.1481862

[7] Brady E., Idris, a general-purpose dependently typed programming language: design and implementation, Journal of Functional Programming, 2013, 23(5), 552-593, DOI: 10.1017/S095679681300018X

[8] Haack C., Wells J. B., Type error slicing in implicitly typed higherorder languages, Science of Computer Programming, Special Issue: 12th European Symposium on Programming (ESOP 2003), 2004, 50(1-3), 189-224, DOI: 10.1016/j.scico.2004.01.004

[9] Zhang D., Myers A. C., Vytiniotis D., Peyton-Jones S., SHErrLoc: a static holistic error locator, ACM Trans. Program. Lang. Syst., 2017, 39(4), Article 18, DOI: 10.1145/3121137

[10] Yang J., Michaelson G., Trinder P., Wells J. B., Improved type error reporting, In: Proceedings of 12th International Workshop on Implementation of Functional Languages, 2000, 71-86

[11] /u/GNULinuxProgrammer, /r/agda: $\mathrm{R} x \rightarrow \mathrm{x}==\mathrm{y} \rightarrow \mathrm{r}$ y, 2016, https://www.reddit.com/r/agda/comments/7lq44q/r x_x_y_r_y/ (Accessed: 2018-06-14)

[12] Watkins K., Cervesato I., Pfenning F., Walker D., A concurrent logical framework I: Judgments and properties, Technical Report CMU-CS-02-101, 2003, https://www.cs.cmu.edu/ fp/ papers/CMU-CS-02-101.pdf

[13] Martin-Löf P., About models for intuitionistic type theories and the notion of definitional equality, in: Proceedings of the Third Scandinavian Logic Symposium, Studies in Logic and the Foundations of Mathematics, North-Holland Publishing Company, Amsterdam, Netherlands, Elsevier, New York, USA, 1975, 82, 81-109, DOI: 10.1016/S0049-237X(08)70727-4

[14] Miller D., Unification under a mixed prefix, J. Symb. Comput., 1992, 14(4), 321-358, DOI: 10.1016/0747-7171(92)90011-R

[15] Abel A., Pientka B., Higher-order dynamic pattern unification for dependent types and records, Typed Lambda Calculi and
Applications, 10th International Conference (Novi Sad, Serbia), Springer Berlin Heidelberg, Berlin, Heidelberg, 2011, 10-26, DOI: 10.1007/978-3-642-21691-6_5

[16] Norell U., Towards a practical programming language based on dependent type theory, Ph.D. thesis, Chalmers University of Technology, Gothenburg, Sweden, 2007, http://www.cse. chalmers.se/ ulfn/papers/thesis.pdf

[17] Cervesato I., Pfenning F., A linear spine calculus, Journal of Logic and Computation, 2003, 13(5), 639-688, DOI: 10.1093/logcom/13.5.639

[18] Harper R., Honsell F., Plotkin G., A framework for defining logics, J. ACM, 1993, 40(1), 143-184, DOI: 10.1145/138027.138060

[19] Ziliani B., Sozeau M., A comprehensible guide to a new unifier for $\mathrm{CIC}$ including universe polymorphism and overloading, Journal of Functional Programming, 2017, 27, e10, DOI: $10.1017 /$ S0956796817000028

[20] Löh A., McBride C., Swierstra W., A tutorial implementation of a dependently typed lambda calculus, Fundamenta Informaticae, Special Issue on Dependently Typed Programming, 2010, 102(2), 177-207, DOI: 10.3233/FI-2010-304

[21] Gundry A. M., Type inference, Haskell and dependent types, Ph.D. thesis, University of Strathclyde, Glasgow, Scotland, United Kingdom, 2013, http://adam.gundry.co.uk/pub/thesis/ thesis-2013-12-03.pdf

[22] Gundry A., McBride C., A tutorial implementation of dynamic pattern unification, Unpublished draft, http://adam.gundry. co.uk/pub/pattern-unify/pattern-unification-2012-07-10.pdf, 2013

[23] Cockx J., Devriese D., Proof-relevant unification: Dependent pattern matching with only the axioms of your type theory, Journal of Functional Programming, 2018, 28, e12, DOI: 10.1017/S095679681800014X

[24] Chen S., Erwig M., Walkingshaw E., An error-tolerant type system for variational lambda calculus, in: Proceedings of the 17th ACM SIGPLAN International Conference on Functional Programming (ICFP '12), ACM, New York, NY, USA, 2012, 29-40, DOI: $10.1145 / 2364527.2364535$

[25] Erwig M., Walkingshaw E., The choice calculus: A representation for software variation, ACM Trans. Softw. Eng. Methodol., 2011, 21(1), Article 6, DOI: 10.1145/2063239.2063245

[26] Chen S., Erwig M., Walkingshaw E., Extending type inference to variational programs, ACM Trans. Program. Lang. Syst., 2014, 36(1), Article 1, DOI: $10.1145 / 2518190$

[27] Eremondi J., Github repository: lambda-pi-constraint, tag thesis-final, 2016, https://github.com/JoeyEremondi/lambdapi-constraint

[28] Zhang D., Myers A. C., Vytiniotis D., Peyton-Jones S., Diagnosing type errors with class, In: Proceedings of the 36th ACM SIGPLAN Conference on Programming Language Design and Implementation (PLDI 2015), ACM, New York, NY, USA, 2015, 12-21, DOI: 10.1145/2737924.2738009

[29] Huet G. P., The undecidability of unification in third order logic, Information and Control, 1973, 22(3), 257-267, DOI: 10.1016/S0019-9958(73)90301-X

[30] Huet G., A unification algorithm for typed $\lambda$-calculus, Theoretical Computer Science, 1975, 1(1), 27-57, DOI: 10.1016/03043975(75)90011-0

[31] Cockx J., Devriese D., Piessens F., Pattern matching without K, In: Proceedings of the 19th ACM SIGPLAN International Conference on Functional Programming (ICFP '14), ACM, New York, NY, 
USA, 2014, 257-268, DOI: 10.1145/2628136.2628139

[32] Cockx J., Devriese D., Piessens F., Unifiers as equivalences: Proof-relevant unification of dependently typed data, In: Proceedings of the 21st ACM SIGPLAN International Conference on Functional Programming (ICFP 2016), ACM, New York, NY, USA, 2016, 270-283, DOI: 10.1145/2951913.2951917

[33] Eremondi J., Improving error messages for dependent types with constraint-based unification, Master's thesis, Utrecht University, Utrecht, Netherlands, 2016, https://dspace.library.uu. $\mathrm{nl} /$ handle/1874/337692

[34] Campora J. P., Chen S., Erwig M., Walkingshaw E., Migrating gradual types, Proceedings of the ACM on Programming Languages, 2018, 2(POPL), Article 15, DOI: $10.1145 / 3158103$
[35] Milner R., A theory of type polymorphism in programming, Journal of Computer and System Sciences, 1978, 17(3), 348-375, DOI: 10.1016/0022-0000(78)90014-4

[36] Hage J., Heeren B., Strategies for solving constraints in type and effect systems, Electronic Notes in Theoretical Computer Science, 2009, 236, 163-183, DOI:10.1016/j.entcs.2009.03.021

[37] Helium Team, Helium github repository, https://github.com/ Helium4Haskell/helium, 2017

[38] Pavlinovic Z., King T., Wies T., Practical SMT-based type error localization, In: Proceedings of the 20th ACM SIGPLAN International Conference on Functional Programming (ICFP'15), ACM, New York, NY, USA, 2015, 412-423, DOI: 10.1145/2784731.2784765 